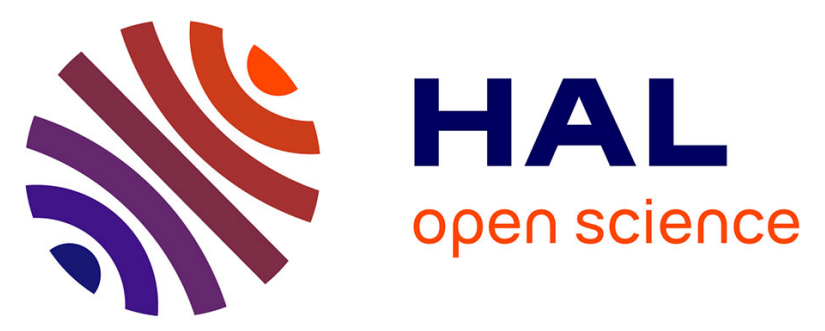

\title{
Experimental validation of a Lyapunov-based controller for the plasma safety factor and plasma pressure in the TCV tokamak
}

Bojan Mavkov, Emmanuel Witrant, Christophe Prieur, E Maljaars, F Felici, O Sauter

\section{To cite this version:}

Bojan Mavkov, Emmanuel Witrant, Christophe Prieur, E Maljaars, F Felici, et al.. Experimental validation of a Lyapunov-based controller for the plasma safety factor and plasma pressure in the TCV tokamak. Nuclear Fusion, 2018, 58 (5), 10.1088/1741-4326/aab16a . hal-01815814

\author{
HAL Id: hal-01815814 \\ https://hal.science/hal-01815814
}

Submitted on 14 Jun 2018

HAL is a multi-disciplinary open access archive for the deposit and dissemination of scientific research documents, whether they are published or not. The documents may come from teaching and research institutions in France or abroad, or from public or private research centers.
L'archive ouverte pluridisciplinaire HAL, est destinée au dépôt et à la diffusion de documents scientifiques de niveau recherche, publiés ou non, émanant des établissements d'enseignement et de recherche français ou étrangers, des laboratoires publics ou privés. 


\title{
Experimental validation of a Lyapunov-based controller for the plasma safety factor and plasma pressure in the TCV tokamak
}

\author{
B. Mavkov ${ }^{1}{ }^{2}$, E. Witrant ${ }^{1}$, C. Prieur ${ }^{1}$, E. Maljaars ${ }^{3}$, F. \\ Felici $^{3}$, O. Sauter ${ }^{4}$ and the TCV-team ${ }^{4}$ \\ ${ }^{1}$ Univ. Grenoble Alpes, CNRS, GIPSA-lab, F-38000 Grenoble, France \\ 2 Department of Electrical and Electronic Engineering, University of Melbourne, \\ Australia \\ ${ }^{3}$ Eindhoven University of Technology, Mechanical Engineering, Control Systems \\ Technology, P.O. Box 513, 5600MB Eindhoven, The Netherlands \\ ${ }^{4}$ SPC-EPFL, CH-1015 Lausanne, Switzerland \\ E-mail: bojan.mavkov@unimelb.edu.au
}

\begin{abstract}
In this paper model-based closed-loop algorithms are derived for distributed control of the inverse of the safety factor profile and the plasma pressure parameter $\beta$ of the TCV tokamak. The simultaneous control of the two plasma quantities is performed by combining two different control methods. The control design of the plasma safety factor is based on an infinite-dimensional setting using Lyapunov analysis for partial differential equations, while the control of the plasma pressure parameter is designed using control techniques for singleinput and single-output systems. The performance and robustness of the proposed controller is analyzed in simulations using the fast plasma transport simulator RAPTOR. The control is then implemented and tested in experiments in TCV Lmode discharges using the RAPTOR model predicted estimates for the $q$-profile. The distributed control in TCV is performed using one co-current and one countercurrent Electron Cyclotron Heating actuation.
\end{abstract}

Keywords: TCV, safety factor, plasma pressure, control, RAPTOR

Submitted to: Nucl. Fusion

\section{Introduction}

One of the main challenges in tokamak plasma control is to achieve the advanced tokamak steady-state scenarios [1]. These scenarios are characterized by a high fusion gain, good plasma confinement and MHD stability. Real-time simultaneous control of several radially distributed magnetic and kinetic plasma parameters is one of the main challenges in the control of hybrid and advanced tokamak scenarios [2]. In this work we are focusing on developing algorithms for simultaneous closed-loop control of the safety factor profile $q$ (and its inverse $\iota$ ) and the plasma pressure or stored energy. Using feedback control in the real-time control system of a tokamak provides 
a better disturbance rejection and less dependency on model uncertainties than using open-loop control only. Feedback control thus improves the performance of reference profile tracking and the robustness of the plasma with respect to MHD instabilities.

Feedback control algorithms for the safety factor can be developed using finite or infinite dimensional feedback control theory. The finite-dimensional control model is obtained by spatial discretization of an infinite-dimensional Partial Differential Equation describing the plasma state. This approach is called an early lumping approach, in which the PDEs are approximated (lumped) first as a set of Ordinary Differential Equations (ODEs) and the control is designed based on this set. Control algorithms for the safety factor profile based on a Multiple Inputs Multiple Outputs (MIMO) approach using different finite dimensional models are developed in previous works [3-9]. There are several works already dedicated to the simultaneous control of the safety factor profile and the pressure parameters. In [10] successful closedloop experiments are performed for the simultaneous control of the relative internal poloidal flux profile $\Psi_{r}$ together with the normalized pressure parameter, $\beta_{N}$. In that work the control-oriented model is obtained from experimental data using a generic two time-scales method. Simultaneous control of the $q$-profile and $\beta_{N}$ using firstprinciples-driven physics-based model is developed for DIII-D H-mode scenarios in [11] and for ITER H-mode scenarios in [12, 13]. The control models used in these works are spatially discretized.

An infinite-dimensional control system is a dynamical system whose state lies in an infinite dimensional vector space, typically the state space of a PDE. In this control approach, the distributed-parameter nature of the system is kept as long as possible in the control design (late lumping approach). This control approach allows us to design feedback control strategies for the full profile throughout the plasma radius.

Control algorithms for the safety factor profile based on infinite dimensional control theory using Lyapunov techniques are already used in several works. Lyapunov functions are energy-like functions that can be used to evaluate the stability of a dynamical system and to design stabilizing controllers. In [14], a strict Lyapunov control function for the diffusion equation of the poloidal magnetic flux gradient is computed. In [15], a Lyapunov-based control strategy using sum-of-squares polynomials is used to maximize the bootstrap current considering a resistivity bounded between two extremal profiles. In [16] a proportional integral (PI) controller is developed for $\mathcal{H} \infty$ stabilization of the spatial distribution of the internal poloidal flux profile $\Psi_{r}$. All these algorithms were tested using only plasma simulators and they were based on the configuration of the Tore Supra tokamak. Tore Supra has circular cross-section and the magnetic flux equation is simplified using cylindrical coordinates. More results using distributed and boundary infinite dimensional control based on IDA-PBC (Interconnexion Damping Assignment- Passive Based Control) design are presented in [17].

The control model is a coupled system of a $1 \mathrm{D}$ resistive diffusion equation $(\iota$ control) and a nonlinear ordinary differential equation ( $\beta$ control). Enabled by the different time scales of the kinetic and magnetic parameters, the control in this work is synthesized by designing separate control algorithms for each controlled variable. The safety factor profile is controlled using the poloidal magnetic flux radial gradient $z$ as a state variable. This parameter is closely related to the safety factor. Our $q$-profile controller is based on an infinite dimensional setting using Lyapunov techniques. We extend the control computation and the stability analysis presented in $[14,18]$ to a more general case without using the cylindrical approximation. The control Lyapunov 
function presented in these works is analogous with the proportional action presented here. Moreover, for successful tracking of the desired reference profiles in the real TCV experiments, an integral action is added in the control design. We have also constrained the shape of the weights in the Lyapunov function to highlight the different spatial locations at which we want to emphasize our control action. The control of the plasma pressure parameter is designed separately using control techniques for Single-Input and Single-Output (SISO) systems.

The control strategy is applied to a controller test environment on TCV L-mode discharges, described in details in [19]. The purpose of this environment is to facilitate offline development and testing of controllers as well as experimental implementation and evaluation on TCV. The fast plasma transport simulator RAPTOR [20, 21] and various algorithms reconstruct the plasma equilibrium and plasma profiles by merging the available measurements with model-based predictions. It estimates the plasma states at many locations, that are provided to the controllers. RAPTOR can also be used as a nonlinear plasma simulator for closed loop control when it is run off-line outside the TCV control system [22]. This enables to test the control algorithms performance and robustness before they are implemented on real TCV experiments.

The paper is organized as follows. In Section 2 the control models and control problems are defined. In Section 3 the control algorithms are presented. The results from the RAPTOR simulations are presented in Section 4 and the results from the TCV experiments are presented in Section 5. Some concluding remarks are given in Section 6 .

\section{Control model and experimental settings}

\subsection{Safety factor control model}

One of the key parameters to analyze the plasma stability and performance is the safety factor $q$. Another parameter that can be used for the control is the inverse of the safety factor $\iota=1 / q$, which can be considered as a more natural control variable since it is proportional to the spatial derivative of the poloidal magnetic flux, whose evolution is governed by a parabolic equation. The corresponding model is an approximate 1D diffusion equation as in [23] assuming constant toroidal magnetic flux at the plasma boundary:

$$
\frac{\partial \psi}{\partial t}=\frac{\eta_{\|} F^{2}}{16 \pi^{2} \mu_{0} \Phi_{b}^{2} \rho} \frac{\partial}{\partial \rho}\left(\frac{g_{2} g_{3}}{\rho} \frac{\partial \psi}{\partial \rho}\right)-\frac{\eta_{\|} B_{0}}{2 \Phi_{b} \rho} V^{\prime}\left(j_{a u x}(u)+j_{b s}\right)
$$

where $\rho$ is the normalized square-root toroidal flux, defined as: $\rho=\sqrt{\frac{\Phi}{\Phi_{b}}}$ ( $\Phi$ being the toroidal magnetic flux and $\Phi_{b}$ its value at the last closed magnetic surface), $B_{0}$ is the magnetic field at the major plasma radius $R=R_{0}, \mu_{0}$ is the permeability of vacuum, $V$ is the plasma volume and $V^{\prime}=\frac{\partial V}{\partial \rho}$ is the spatial derivative of the plasma volume, and $\eta_{\|}$is the parallel electrical neoclassical resistivity of the plasma. The non-inductive current density $j_{n i}=j_{a u x}+j_{b s}$ is obtained by combining the auxiliary sources of current density $j_{a u x}(u)$ (heating and current drive radio-frequency (RF) systems) and the bootstrap current density $j_{b s}$. The vector $u$ contains the actuator inputs represented by the power delivered by the Electron-Cyclotron (EC) clusters $\left[P_{E C, 1}(t) ; P_{E C, 2}(t)\right]$ used in the RAPTOR simulations and in the TCV experiments. 
The coefficients $F, g_{2}, g_{3}$ and $V^{\prime}$ are defined as $[24,25]$ :

$$
F=R B_{\phi} ; \quad g_{2}=\left(\frac{\partial V}{\partial \psi}\right)\left\langle\frac{|\nabla \psi|}{R^{2}}\right\rangle ; \quad g_{3}=\left\langle\frac{1}{R^{2}}\right\rangle ; \quad V^{\prime}=\frac{\partial V}{\partial \rho}
$$

where $\langle\cdot\rangle$ denotes a flux surface average, $R$ is the distance from the vertical axis and $B_{\phi}$ is the toroidal magnetic field.

In this configuration, the Electron Cyclotron Current Drive System (ECCD) is represented by co-current (with respect to the ohmic plasma current) and countercurrent deposition. The gyrotrons in TCV are grouped in clusters. The power delivered by the first cluster (with input power $P_{E C, 1}(t)$ ) consists of one gyrotron with counter-current drive. The second cluster (with input power $P_{E C, 2}(t)$ ) consists of two gyrotrons that are used for co-current drive. A set of coils generating a poloidal magnetic field are used for feedback control of the plasma vertical and horizontal position in the poloidal plane. The position of the magnetic field is chosen such that the EC power deposition is absorbed on the magnetic axis. The total auxiliary current density model is computed as the sum of the current densities induced by each ECCD antenna and the bootstrap current density: $j_{\mathrm{ni}, \bmod }=\sum_{1}^{n_{\mathrm{aux}}} j_{\mathrm{aux}, i}+j_{b s}$, where $n_{\text {aux }}=2$ denotes the number of EC antennas used in our case. Each individual auxiliary current drive is modeled by the scaling law presented in [22]:

$$
j_{\mathrm{aux}, i}(\rho, t)=\frac{T_{e}}{n_{e}} j_{\mathrm{dis}, i}(\rho) P_{\mathrm{EC}, i}(t)
$$

which is the product of the weighted Gaussian distributions $j_{\text {dis }, i}(\rho)$ (given in [20]) with the input powers $P_{\mathrm{EC}, i}(t)$ and the current-drive efficiency $\left(\frac{\bar{T}_{e}}{n_{e}}\right)$. The nominal current deposition width $\omega_{\text {dep }}=0.35$ and the nominal location of the peak of the deposition $\rho_{\text {dep }}=0$ are used for both clusters. The reference values of $j_{\text {dis }}(\rho)$ and the relative power density profile $Q_{d i s}(\rho)$ for the two EC clusters are shown in Fig. 1.

The bootstrap current profile $j_{b s}$ and the neoclassical resistivity $\eta_{\|}$models are the one
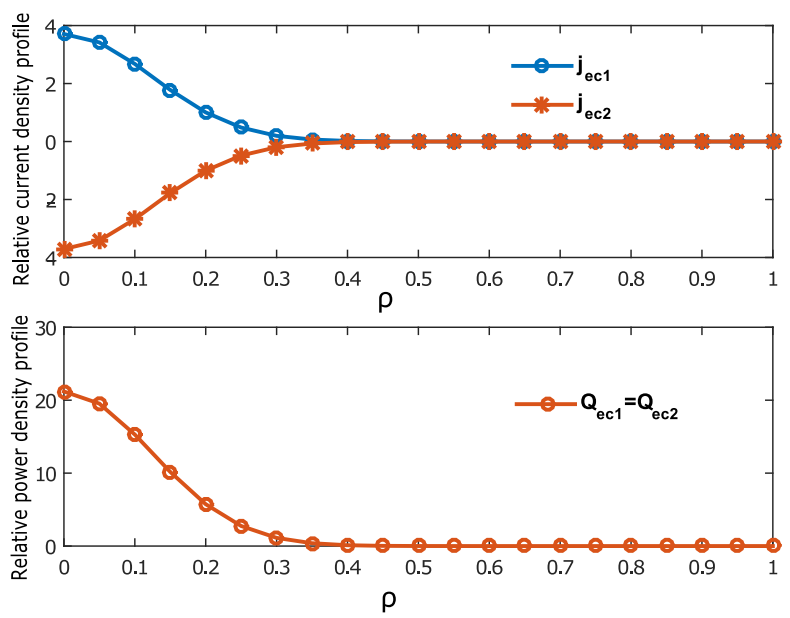

Figure 1: The relative current density profile $j_{\text {dis,ec }}\left(10^{20} \frac{\mathrm{A}}{\mathrm{m}^{5} \mathrm{keV} \mathrm{W}}\right)$ and relative power density profile $Q_{d i s, e c}\left(\mathrm{~m}^{-3}\right)$ for the TCV configuration.

used in the RAPTOR code and are based on the Sauter-Angioni equations [26, 27]. 
The model of the bootstrap current density can be used in a simplified version under the assumption of a tight coupling between the electron and ion species. With this assumption the electron and the ion densities are considered to be equal $n_{e}=n_{i}$ and $\frac{\partial \ln \left(T_{i}\right)}{\partial \Phi}=\frac{\partial \ln \left(T_{e}\right)}{\partial \Phi}$, where $T_{i}$ is the ion temperature. The approximated model is derived as :

$$
j_{b s}=\frac{k_{b s}}{\partial \Psi / \partial \rho}\left[\mathcal{L}_{31} \frac{\partial n_{e}}{\partial \rho} T_{e}+\left(\mathcal{L}_{31}+R_{p e} \mathcal{L}_{32}+\left(1-R_{p e}\right) \mathcal{L}_{34}\right) \frac{\partial T_{e}}{\partial \rho} n_{e}\right]
$$

where $k_{b s}, \mathcal{L}_{31}, \mathcal{L}_{32}, \mathcal{L}_{34}$ depend on magnetic configuration of the plasma equilibrium and $R_{p e}=p_{e} / p$ is the ratio between electrons and total pressures.

The neoclassical electrical resistivity model in RAPTOR is calculated as:

$$
\eta_{\|}=1 / \sigma_{\|}=1 /\left(c_{\text {neo }}(\rho) \sigma_{\text {Spitzer }}\right)
$$

where $\sigma_{\|}$is neoclassical electrical conductivity and $\sigma_{\text {Spitzer }} \propto T_{e}^{3 / 2}$ e is the Spitzer conductivity. The parameter $c_{\text {neo }}$ is the neoclassical correction, which depends on geometric effects as well as collisionality. These two plasma parameters are timevarying and highly dependent on the electron and ion temperatures and densities.

The controlled state is selected to be the magnetic flux gradient $z=\frac{\partial \Psi}{\partial \rho}$. The variables of main interest for the control ( $q, \iota$ and current profiles) can be easily related to the magnetic flux gradient $\iota=\frac{1}{q}=\frac{1}{2 \Phi_{b} \rho} z$. Differentiating (1) in space and considering the previous change of variables, as in [28], yields:

$$
\frac{\partial z}{\partial t}=\frac{\partial}{\partial \rho}\left(\frac{a_{1}(\rho)}{\rho} \frac{\partial}{\partial \rho}\left(a_{2}(\rho) z\right)\right)-\frac{\partial}{\partial \rho}\left(a_{3}(\rho)\left(j_{\text {aux }}(u)+j_{b s}\right)\right)
$$

with boundary conditions:

$$
\begin{aligned}
& z(0, t)=0, \forall t \geq 0 \\
& z(1, t)=a_{4}(1) I_{p}(t), \forall t \geq 0
\end{aligned}
$$

and initial condition:

$$
z(\rho, 0)=z_{0}(\rho), \forall \rho \in[0,1]
$$

where $a_{1}(\rho)=\frac{\eta \| F^{2}}{16 \pi^{2} \mu_{0} \Phi_{b}^{2}}, a_{2}(\rho)=\frac{g_{2} g_{3}}{\rho}, a_{3}(\rho)=\frac{\eta_{\|} B_{0}}{2 \Phi_{b} \rho} V^{\prime}, a_{4}(\rho)=\frac{\rho}{g_{2} g_{3}} \frac{16 \pi^{3} \mu_{0} \Phi_{b}}{F}$ and $I_{p}$ is the total plasma current.

For control purposes we linearize the model around a given equilibrium profile $\bar{z}$. This equilibrium is calculated by taking the values of the plasma parameters when the inputs $\left(\bar{u}, \bar{I}_{p}\right)$ are applied sufficiently long for the system to reach a steady state:

$$
0=\frac{\partial}{\partial \rho}\left(\frac{a_{1}(\rho)}{\rho} \frac{\partial}{\partial \rho}\left(a_{2}(\rho) \bar{z}\right)\right)-\frac{\partial}{\partial \rho}\left(a_{3}(\rho)\left(\bar{j}_{a u x}(\bar{u})+\bar{j}_{b s}\right)\right)
$$

with boundary conditions: $\bar{z}(0, t)=0, \bar{z}(1, t)=a_{4}(1) \bar{I}_{p}(t)$. The linearized model is derived around the steady state by substituting, in the reference model, $z=\bar{z}+\tilde{z}$, $u=\bar{u}+\tilde{u}$ and $I_{p}=\bar{I}_{p}+\tilde{I}_{p}$ using Taylor series with first order approximation. Here $\left(\bar{z}, \bar{u}, \bar{I}_{P}\right)$ denotes the equilibrium of the system and $\left(\tilde{z}, \tilde{u}, \tilde{I}_{p}\right)$ denotes the variations around this equilibrium. The simplified linearized model obtained from (6) is derived as:

$$
\frac{\partial \tilde{z}}{\partial t}=\frac{\partial}{\partial \rho}\left(\frac{a_{1}(\rho)}{\rho} \frac{\partial}{\partial \rho}\left(a_{2}(\rho) \tilde{z}\right)\right)-\frac{\partial}{\partial \rho}\left(a_{3}(\rho)\left(\tilde{j}_{a u x}(\tilde{u})+\tilde{j}_{b s}\right)\right)
$$




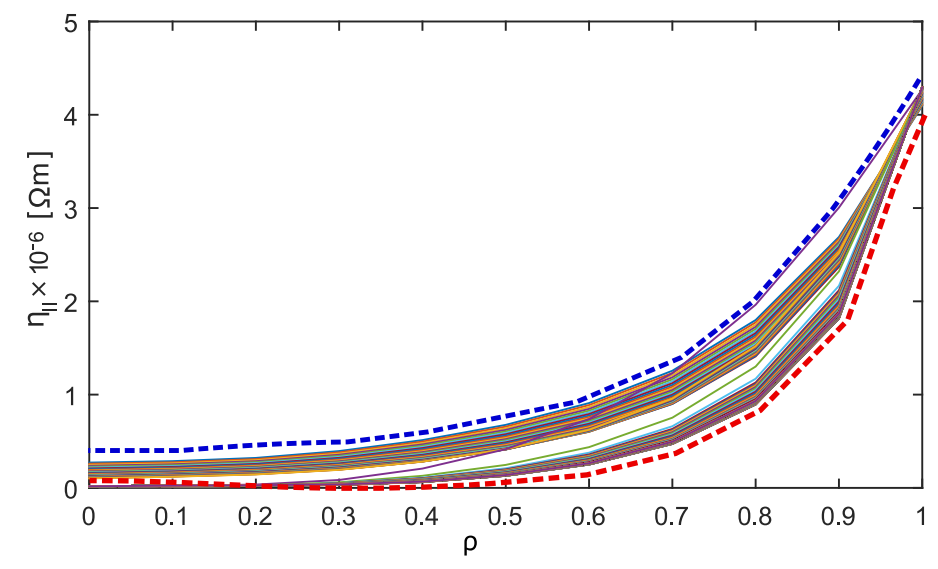

Figure 2: $\eta_{\|}$parameter ranges with its minimum (red dashed line) and its maximum value (blue dashed line).

with boundary conditions:

$$
\begin{aligned}
& \tilde{z}(0, t)=0, \forall t \geq 0 \\
& \tilde{z}(1, t)=a_{4}(1) \tilde{I}_{p}(t), \forall t \geq 0
\end{aligned}
$$

and initial condition:

$$
\tilde{z}\left(\rho, t_{0}\right)=\tilde{z}_{0}, \forall \rho \in[0,1]
$$

For the control application, the perturbed bootstrap current $\tilde{j}_{b s}(\rho, t)=j_{b s}-\bar{j}_{b s}$ can be considered as an external disturbance to be attenuated/compensated (since $I_{b s} / I_{p}<=10 \%$ in L-mode plasmas). When the perturbed bootstrap current is relatively large and cannot be neglected, it should be included in the stability analysis. Infinite dimensional feedback control strategies, where the bootstrap current is included in the control design and stability analysis can be found in [15, 29]. The coefficient $\bar{\eta}_{\|}$can be considered as an uncertain parameter that varies in a given operational range:

$$
\bar{\eta}_{\|}(\rho, t)=\omega_{\eta}(t) \eta_{\|, \min }(\rho)+\left(1-\omega_{\eta}(t)\right) \eta_{\|, \max }(\rho), \text { for all } t \geq 0
$$

where $\omega_{\eta}(t) \in[0,1]$ and $\eta_{\|, \text {min }}$ and $\eta_{\|, \text {max }}$ are the minimum and the maximum values of $\eta_{\|}$, respectively, presented in Fig. 2. The bounds of the resistivity $\eta_{\|}$are calculated using the model (5), by modulating the ECCD clusters with different power intensities in the RAPTOR simulations. From the plot in Fig. 2 we can see that the variations of $\eta_{\|}$are not significant in the operation range that is considered in our experiments. We thus assume that the $\eta_{\|}$profile scales linearly between the two outer bounds $\left(\eta_{\|, \text {min }}\right.$ and $\left.\eta_{\|, \max }\right)$. While this uncertainty is not crucial for the control of a L-mode plasma, it may become important in other plasma operation modes in which the plasma resistivity changes more dramatically. 


\subsection{Plasma pressure and thermal energy control model}

The plasma parameter $\beta$ is defined as the ratio between the volume-averaged pressure and the magnetic pressure. This parameter is of prime interest to analyze MHD stability in tokamaks. There are several definitions of this parameter. Following [30], the most commonly used one is:

$$
\beta=\frac{\langle p\rangle_{V}}{B_{0}^{2} /\left(2 \mu_{0}\right)}=\frac{2}{3} \frac{W_{t h} / V}{B_{0}^{2} /\left(2 \mu_{0}\right)}
$$

where $W_{t h}$ is the stored thermal energy in the plasma and $\langle\cdot\rangle_{V}$ denotes the volumeaverage operation $1 / V \int_{V}(\cdot) d V$. The energy stored in the plasma is presented by [28]:

$$
W_{t h}=\frac{3}{2} \int_{V}\left(n_{e} T_{e}+n_{i} T_{i}\right) d V
$$

where $T_{i}$ is the ions temperature profile and $n_{i}$ is the ions density profile. Assuming that the plasma volume does not change in time, the approximate energy balance equation is given by:

$$
\frac{d W_{t h}}{d t}=-\frac{W_{t h}}{\tau_{t h}}+P_{\mathrm{tot}}(u)
$$

where $\tau_{t h}$ is the global energy confinement time which can be modelled using a scaling law $[31,32]$ such as $\tau_{t h}=0.14 I_{p}^{0.91} B_{0}^{-0.13} n_{e}^{-0.77} P_{t o t}^{-0.75}$, and the total power $P_{\text {tot }}(u)$ can be derived as a sum of several contributions. The main sources of electrons heating are the auxiliary radio frequency (RF) heating sources. Other sources are the ohmic heating power, electron-ion power loss and radiation power loss. All these terms depend on many plasma parameters (temperature, $q$, etc.) as well as their radial derivative (e.g. temperature gradients, magnetic shear, etc.).

The simplified linearized model of (16) around the steady state $\left(\bar{W}_{t h}, \bar{u}, \bar{I}_{p}\right)$ is derived using Taylor series with first order approximation considering the model of $\tau_{t h}$ given in [31]:

$$
\begin{aligned}
\frac{d \tilde{W}_{t h}}{d t} & =-\frac{\tilde{W}_{t h}}{\bar{\tau}_{t h}}+k_{p} \tilde{P}_{t o t}(\tilde{u})+k_{I p} \tilde{I}_{p}+\omega \\
\tilde{\beta} & =\frac{2}{3} \frac{\tilde{W}_{t h} / V}{B_{0}^{2} /\left(2 \mu_{0}\right)}
\end{aligned}
$$

where $k_{I p}=-\frac{\partial\left(\bar{W}_{t h} / \bar{\tau}_{t h}\right)}{\partial \bar{I}_{p}}+\frac{\partial \bar{P}_{t o t}}{\partial \bar{I}_{P}}, k_{p}=-\frac{\partial\left(\bar{W}_{t h} / \bar{\tau}_{t h}\right)}{\partial \bar{P}_{t o t}}+1$, and $\omega$ contains the variations of $\tau_{t h}$ that depend on plasma parameters other then $P_{t o t}$ and $I_{p}$ (considered as disturbances in the system, i.e. the variation of the ohmic power). The parameter $\bar{\tau}_{t h}$ is assumed to be bounded as $\bar{\tau}_{\text {min }} \leq \bar{\tau}_{t h} \leq \bar{\tau}_{\text {max }}$. In our simplified control model, the total heating power is calculated as the sum of the two main sources of heating $\tilde{P}_{\text {tot }}(\tilde{u})=\tilde{P}_{E C, 1}(t)+\tilde{P}_{E C, 2}(t)$.

Although there is a coupling between the dynamics of these two states $\left(\tilde{z}\right.$ and $\left.\tilde{W}_{t h}\right)$, the control actions are computed separately. Variations of the other plasma parameters are considered as disturbances that need to be handled by the robustness margin of the controller. The ion and electron temperatures and densities are considered as uncertainties for $\iota$ (leading to the bounds on $\eta_{\|}$in Fig. 2) or volume-averaged for $\beta$. The profiles distributions and couplings, e.g between $q$ and $T_{e}$, can be considered with more complex PDE controllers as proposed in [29] but we focus here on the control design motivated by the experimental setup. 


\section{Feedback control design}

In this section we present the control algorithms that were implemented in the TCV experimental session on feedback controllers testing in 2016/2017, in which several different controllers were implemented $[19,33,34]$. In these TCV experiments only the powers of the two (ECH/ECCD) antennas are used as in-domain control actuators. The control algorithms are designed separately for $\iota$ and $\beta$, and a composite control of two signals, $u_{\iota}$ for the control of $\iota$ and $u_{\beta}$ for control of $\beta$, is sent to the TCV actuators. For the control of the $\iota$ profile a proportional-integral infinite dimensional state feedback control is applied and the control of the $\beta$ parameter is tuned using classical control methods for ODEs. An anti-windup compensator is added to the control design to avoid the drawbacks from the actuators saturation in the system (discussed in details in Appendix C). The complete control scheme is presented as a block diagram in Fig. 3.

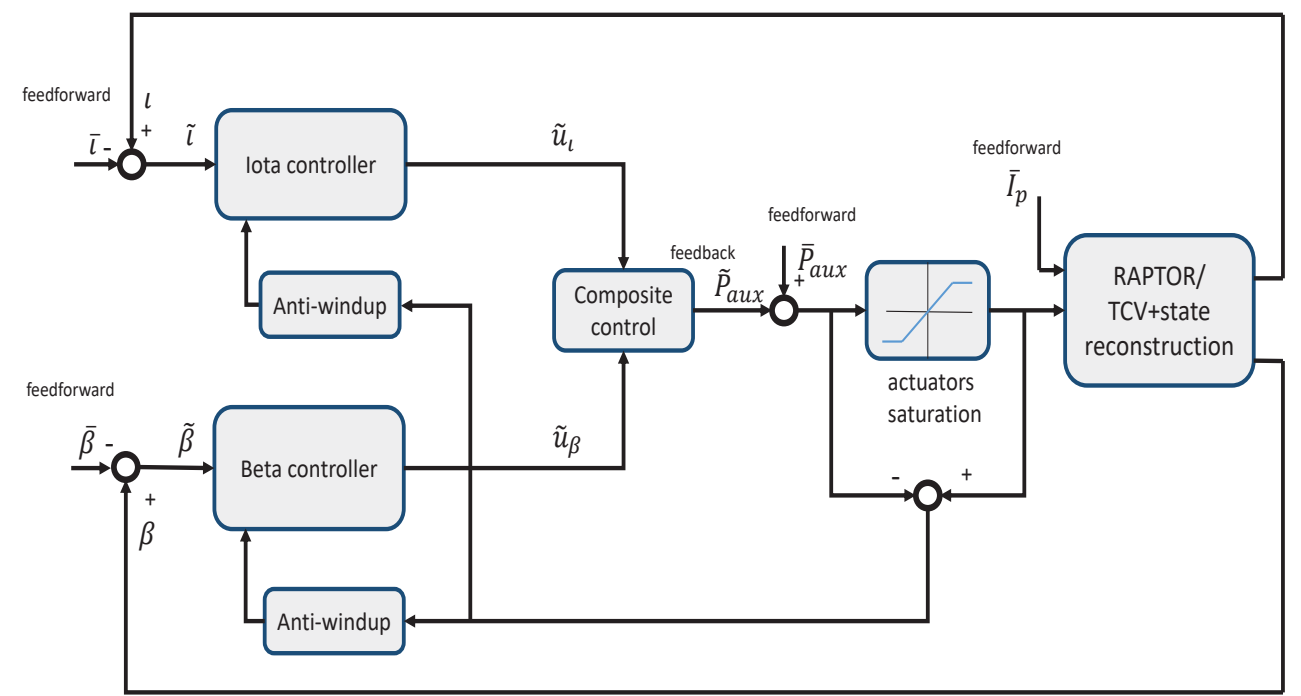

Figure 3: Control scheme

\subsection{Distributed control of $\iota$}

The feedback control of the $\iota$ profile is achieved with an infinite dimensional PI controller. The auxiliary heating powers are set to obtain the desired profile of $\tilde{j}_{n i}$. The feedback control is applied using the real-time estimate of $\tilde{z}$, which represents the error between the $z$ profile and the desired reference profile $\bar{z}$. The feed-forward signal corresponding to this stationary profile is calculated by (9) and is set with $\bar{u}$ and $\bar{I}_{P}$. In the simulation results the feedworward term $\bar{z}$ is directly obtained from RAPTOR as steady-state (along with $\bar{u}$ and $\bar{I}_{p}$ ), while in the TCV experiments the feedforward term is calculated by numerically solving $\bar{u}$ and $\bar{I}_{p}$ from the equality (9) with the given values of the desired target profile $\bar{z}$. 
In Lyapunov-based control, the control law is designed such that closed-loop stability can be guaranteed using Lyapunov stability analysis. Lyapunov functions are energy-like functions and by examining their time derivative we can conclude that the closed-loop system is stable or asymptotically stable [35]. The asymptotic stability of the closed-loop system guarantees that the system converges to the desired equilibrium. The stability analysis of the closed-loop system will be discussed in Section 3.2. To obtain an appropriate closed-loop system description, the control law is written in a specific form. For this purpose, the feedback-control of the $z$-profile is determined such that the control parameter $\tilde{j}_{n i, f b}$ is calculated as:

$$
\tilde{j}_{n i, f b}\left(\tilde{u}_{\iota}, \rho, t\right)=\frac{1}{a_{3}(\rho)}\left(\int_{0}^{\rho} \alpha_{p}(r) \tilde{z}(r, t) d r-\int_{0}^{\rho} \mathcal{I}_{\iota}(r, t) d r\right)
$$

where the proportional action is represented by weighted spatial integral of the error on the $z$ profile $(\tilde{z})$ and the integral action of the control is calculated as:

$$
\frac{\partial \mathcal{I}_{\iota}(\rho, t)}{\partial t}=-\alpha_{I}(\rho) \tilde{z}(\rho, t)-\lambda(t) \mathcal{I}_{\iota}(\rho, t)
$$

where $\tilde{u}_{\iota}$ stands for the feedback control signal for the $\iota$ parameter, $\mathcal{I}_{\iota}$ is the integral action of the controller, and $\alpha_{p}(\rho)$ and $\alpha_{I}(\rho)$ correspond to the proportional and the integral gain of the control, respectively. The implementation of the feedback control is discussed in Section 3.4.

The parameter $\lambda(t) \in\left[0, \lambda_{\max }\right]$ (for a given $\lambda_{\max }$ ) in the integral action is called a "forgetting factor" for the integrator $[4,36]$. This parameter is used to cancel high overshoots by weighting down past accumulated errors when $\iota$ is far from the reference profile. This usually appears when the target profile is changed. This term vanishes in finite time to avoid a steady-state error $(\lambda(t) \rightarrow 0$ when $t \rightarrow \infty)$ and is activated at the beginning of the control or at the time instants when there are sudden (significant) changes of the reference profile. The value of this parameter is selected as $\lambda(t)=\kappa e^{-\sigma t^{2}}$, where $\kappa$ and $\sigma$ are chosen constant parameters. The values of these parameters are manually tuned to obtain the best performance of the controller.

The resulting closed-loop system (10), when the feedback control (18) applies, has the dynamics:

$$
\left\{\begin{array}{l}
\frac{\partial \tilde{z}}{\partial t}=\frac{\partial}{\partial \rho}\left(\frac{a_{1}(\rho)}{\rho} \frac{\partial}{\partial \rho}\left(a_{2}(\rho) \tilde{z}\right)\right)-\alpha_{p}(\rho) \tilde{z}+\mathcal{I}_{\iota} \\
\frac{\partial \mathcal{I}}{\partial t}=-\alpha_{I}(\rho) \tilde{z}-\lambda(t) \mathcal{I}_{\iota}
\end{array}\right.
$$

In this TCV experimental setup $I_{p}$ is feedback controlled via the inductive voltage and its value is set to the desired reference value $I_{p}=\bar{I}_{p}$. This leads to the following boundary conditions:

$$
\begin{aligned}
& \tilde{z}(0, t)=0, \forall t \geq 0 \\
& \tilde{z}(1, t)=0, \forall t \geq 0
\end{aligned}
$$

The stability analysis is crucial for the feedback control design and they are used to prove that the closed loop system converges to the desired equilibrium point. This means that the parameters $\alpha_{p}$ and $\alpha_{I}$ of the controller should be chosen such that the closed-loop system is asymptotically stable. 


\subsection{Stability of the closed loop system}

The stability analysis for the closed-loop system (20) is performed using Lyapunov analysis for infinite-dimensional systems. More results on Lyapunov theory for infinite dimensional systems can be found in $[37,38]$. To compute the stability of the closedloop system (20), the following candidate Lyapunov function is chosen:

$$
\mathcal{V}=\mathcal{V}_{z}+\mathcal{V}_{I}
$$

where

$$
\mathcal{V}_{z}=\frac{1}{2} \int_{0}^{1} \rho^{2} p_{z}(\rho) \tilde{z}^{2} d \rho
$$

and

$$
\mathcal{V}_{I}=\frac{1}{2} \int_{0}^{1} \rho^{2} p_{I}(\rho) \mathcal{I}_{\iota}^{2} d \rho
$$

The candidate Lyapunov function is chosen as a weighted $L^{2}(0,1)$ norm $\ddagger$, where $p_{z}(\rho)>0$ and $p_{I}(\rho)>0$ for $\rho \in[0 ; 1]$ are the weighting polynomial functions, and it is multiplied by the term $\rho^{2}$ to handle the singularity at $\rho=0$.

Theorem 1. Suppose that for a given positive value $\gamma_{1}$ and a time-varying positive number $\gamma_{2}(t)$, there exist polynomials $p_{z}, p_{I}, k_{z}=\alpha_{p} p_{z}, k_{I}=\alpha_{I} p_{I}$ such that $p_{z}(\rho)>0$, $p_{I}(\rho)>0, k_{z}>0$ and $k_{I}>0$ for all $\rho \in[0,1]$. If the following inequality is verified:

$$
A(\rho)+A_{1}(\rho, t) \leq 0
$$

where

$$
\begin{gathered}
A(\rho)=\left[\begin{array}{ccc}
c_{1,1}(\rho) & c_{1,2}(\rho) & c_{1,3}(\rho) \\
0 & c_{2,2}(\rho) & 0 \\
c_{1,3}(\rho) & 0 & c_{3,3}(\rho)
\end{array}\right] \\
c_{1,1}=-\left(2 p_{z}(\rho)+\rho p_{z}^{\prime}(\rho)\right) a_{1}(\rho) \frac{\partial\left(a_{2}(\rho)\right)}{\partial \rho}-k_{z}(\rho) \rho^{2} \\
c_{2,2}=-\rho p_{z}(\rho) a_{1}(\rho) a_{2}(\rho) \\
c_{1,2}=-\frac{1}{2}\left(2 p_{z}(\rho) a_{2}(\rho)+\rho p_{z}(\rho) \frac{\partial a_{2}(\rho)}{\partial \rho}+\rho p_{z}^{\prime}(\rho) a_{2}(\rho)\right) a_{1}(\rho) \\
c_{1,3}=\frac{1}{2} \rho^{2}\left(p_{z}(\rho)-k_{I}(\rho)\right) \\
c_{3,3}=-\rho^{2} p_{I}(\rho) \lambda(t)
\end{gathered}
$$

and

$$
A_{1}(\rho, t)=\frac{1}{2}\left[\begin{array}{ccc}
\gamma_{1} p_{z}(\rho) \rho^{2} & 0 & 0 \\
0 & 0 & 0 \\
0 & 0 & \gamma_{2}(t) p_{I}(\rho) \rho^{2}
\end{array}\right]
$$

then the time derivative $\dot{\mathcal{V}}$ of $\mathcal{V}$ defined in (22) along the solutions to (20) and (21) verifies:

$$
\dot{\mathcal{V}} \leq-\frac{1}{2} \gamma_{1} \int_{0}^{1} \rho^{2} p_{z}(\rho) \tilde{z}^{2} d \rho-\frac{1}{2} \gamma_{2}(t) \int_{0}^{1} \rho^{2} p_{I}(\rho) \mathcal{I}_{\iota}^{2} d \rho \leq-\gamma(t) \mathcal{V}
$$

$\ddagger$ The $L^{2}$ norm of $\xi$ on a domain $\Omega$ is denoted as $\|\xi\|_{L^{2}}=\left(\int_{\Omega} \xi^{2} d \Omega\right)^{\frac{1}{2}}$ 
where

$$
\gamma(t)=\min _{t \in[0, T]}\left(\gamma_{1}, \gamma_{2}(t)\right)
$$

The proof of this theorem is given in Appendix A.

The numerical computation of the the polynomial functions $p_{z}(\rho), p_{I}(\rho), k_{z}(\rho)$ and $k_{I}(\rho)$ is presented in Appendix B. Once the solution of the polynomial functions is found, the controller gains are calculated as $\alpha_{p}=k_{z} / p_{z}, \alpha_{I}=k_{I} / p_{I}$.

The stability of our coupled system of linearized equations is established with Theorem 1. The parameters $\gamma_{1}$ and $\gamma_{2}(t)$ determine the convergence rate of our closedloop system. By changing these parameters we can obtain the desired values of $\alpha_{z}(\rho)$ and $\alpha_{I}(\rho)$ to obtain best performance of the controller. The parameter $\gamma_{2}(t)=\kappa_{\gamma} e^{-\sigma t^{2}}$ is selected to be time-varying according to the time-varying parameter $\lambda(t)$. Here $\kappa_{\gamma}$ is a tunning parameter and the term $e^{-\sigma t^{2}}$ is the same one that is used to define $\lambda(t)$.

Another important property is to estimate how quickly the solutions converge to the desired equilibrium state.

Corollary 1. Under the conditions of Theorem 1 and using the definition of $\gamma(t)$, the closed-loop system (20) with boundary condition (21) and initial conditions (12) is globally exponentially stable. The convergence rate of the system satisfies:

$$
\mathcal{V}\left(\tilde{z}, \mathcal{I}_{\iota}\right) \leq e^{-\int_{0}^{t} \gamma(r) d r} \mathcal{V}\left(\tilde{z}_{0}, \mathcal{I}_{\iota, 0}\right)
$$

where $\gamma(t)$ is given in (30).

Proof. Using separation of variables and integrating the inequality (29) over time give the result.

\subsection{Control of $\beta$}

The control synthesis for $\beta$ is developed using only the auxiliary heating sources as the system input, given by $\tilde{P}_{\text {tot }}\left(\tilde{u}_{\beta}\right)$. The value of the plasma current during the experiments is considered as: $I_{p}(t)=\bar{I}_{p}$. The closed-loop control algorithm of $\beta$ is developed using classical methods for designing Proportional-Integral-Derivative (PID) controllers. There are many works dedicated to optimal tuning of the parameters of PID controllers. Such methods can be found in $[39,40]$. In this section the simple rule for PID tuning presented in [41] is used. This rule is called Simple Internal Model Control (SIMC). In our application only Proportional (P) and Integral (I) components are applied, whereas the Derivative (D) component is not necessary to be used when the dynamic of the system is represented by first order ODE. In the TCV control system there can be several sources of time delays [19] (e.g. signal routing delays and reconstruction delays). The effective delay in the TCV experiments can be up to $\theta \approx 5 \mathrm{~ms}$ and this is taken into consideration when the feedback control is designed. The transfer function of the derived PI controller is chosen in cascade form as:

$$
c(s)=\frac{\tilde{P}_{\mathrm{tot}}(s)}{\tilde{\beta}(s)}=-K_{c} \frac{\tau_{I} s+1}{\tau_{I} s}
$$

or presented in time domain:

$$
\tilde{P}_{\text {tot }}\left(\tilde{u}_{\beta}(t)\right)=-K_{c} \tilde{\beta}(t)-\int_{0}^{1} \frac{K_{c}}{\tau_{I}} \tilde{\beta}(\sigma) d \sigma=-K_{\beta, p} \tilde{\beta}(t)-\int_{0}^{t} K_{\beta, i} \tilde{\beta}(\sigma) d \sigma
$$


where $K_{\beta, p}$ is the proportional gain and $K_{\beta, i}$ is the integral gain of the controller. The coefficients of the controller are computed as follows:

$$
\begin{aligned}
K_{c} & =\frac{1}{k_{\beta}} \frac{\bar{\tau}_{t h}}{\tau_{c}+\theta}=\frac{1}{k_{\beta}^{\prime}} \frac{1}{\tau_{c}+\theta} \\
\tau_{I} & =\min \left\{\bar{\tau}_{t h}, \frac{4}{k_{\beta}^{\prime} K_{c}}\right\}=\min \left\{\bar{\tau}_{t h}, 4\left(\tau_{c}+\theta\right)\right\}
\end{aligned}
$$

where $k_{\beta}=k_{p} \bar{\tau}_{t h} \frac{\beta}{W_{t h}}=\frac{2 k_{p} \bar{\tau}_{t h}}{3 V B_{0}^{2} /\left(2 \mu_{0}\right)}$ and $k_{\beta}^{\prime}=\frac{k_{\beta}}{\bar{\tau}_{t h}}$. The SIMC PI-rule has one tuning parameter $\tau_{c}$ which can be used as a trade off between performance ("tight" control) and robustness ("smooth" control). Moreover, the value of this parameter can be changed according to the control requirements. For a robust performance of the tracking, this parameter should be $\tau_{c} \geq \theta$. If this parameter is decreased the convergence speed and the disturbance rejection are improved. For better stability, robustness and small input usage the value of this parameter should be increased. The optimal tuning of this parameter and the performance of the PI SIMC method is discussed in [42].

\subsection{Control implementation}

As stated in the previous section, only the auxiliary powers are used as controlled inputs in the system. This leads to limited shapes of the generated current density profiles due to the limited degrees of freedom of the actuators. An optimization problem should be formulated to fit the desired current density control calculated in (18) with the achievable current density profile. The control is solved such that an optimization problem is formulated to deal with the simultaneous control of several parameters. The control inputs $\tilde{u}=\left[\begin{array}{ll}\tilde{P}_{E C, 1} & \tilde{P}_{E C, 2}\end{array}\right]$ are calculated using an optimization algorithm that solves:

$$
\arg \min _{\tilde{u}} \omega_{1} f_{z}\left(\tilde{u}_{\iota}\right)+\omega_{2} f_{\beta}\left(u_{\beta}\right)
$$

subject to

$$
\begin{aligned}
& \tilde{P}_{1, \min } \leq \tilde{P}_{\mathrm{EC}, 1} \leq \tilde{P}_{1, \max } \\
& \tilde{P}_{2, \min } \leq \tilde{P}_{\mathrm{EC}, 2} \leq \tilde{P}_{2, \max }
\end{aligned}
$$

where:

$$
\begin{aligned}
& f_{z}\left(\tilde{u}_{\iota}\right)=\int_{0}^{1} \frac{1}{\sigma_{j}^{2}}\left(\tilde{j}_{\mathrm{ni}, \mathrm{fb}}-\tilde{j}_{\mathrm{ni}, \bmod }\left(\tilde{u}_{\iota}\right)\right)^{2} d \rho \\
& f_{\beta}\left(\tilde{u}_{\iota}\right)=\frac{1}{\tilde{P}_{t o t, f b}^{2}}\left(\tilde{P}_{t o t, f b}-\tilde{P}_{t o t}\left(\tilde{u}_{\beta}\right)\right)^{2}
\end{aligned}
$$

$\tilde{j}_{\text {ni,fb }}$ is the desired feedback control calculated in (18) and $\tilde{j}_{\text {ni,mod }}\left(\tilde{u}_{\iota}\right)$ is the current profile that can be achieved by the available actuators in this experimental setup. Here $\tilde{P}_{t o t, f b}$ is the desired feedback control calculated in $(34)$ and $\tilde{P}_{t o t}\left(\tilde{u}_{\beta}\right)$ is total power that can be achieved by the available actuators. The parameters required to calculate the desired profile $\tilde{j}_{\text {ni,mod }}$ are provided in real-time by the RAPTOR observer in the TCV experiments. The cost function $f_{z}$ is normalized by multiplying with the 
inverse of the square of the variance in the errors $\sigma_{j}$, that is common technique used in weighted least squares problems, and $f_{\beta}$ is normalized by multiplying with the inverse of $\tilde{P}_{t o t, f b}$.

The parameters $\omega_{1}$ and $\omega_{2}$ are used as scaling parameters in the optimization. They can be used to tune the priority of the control of one of the plasma parameters. The nominal values of these parameter are set as $\omega_{1}=\omega_{2}=0.5$ to give equal priority to both plasma parameters. These weighting parameters can be changed according to the different control objectives (e.g. $\omega_{1}=0, \omega_{2}=1$ to yield only $\beta$ control).

In (35), $\tilde{P}_{\text {tot }}\left(\tilde{u}_{\beta}\right)$ is the desired control of the $\beta$ component calculated in (33).

Remark 1. The global exponential stability of the nominal system for the evolution of $z(\tilde{u}=0$ when $u=\bar{u})$ is proven in several previous works [14, 18, 29, 43]. In this work, when closing the loop (20), the contribution of the feedback control $\left(-\alpha_{p}(\rho) \tilde{z}+\mathcal{I}_{\iota}\right)$ accelerates the convergence to the desired reference. The limitations of the achievable shapes for the auxiliary current deposit can reduce the desired convergence rate. In the worst case, $\tilde{u}=0$ is always a feasible state that results to globally exponentially stable system.

\subsection{Performance criterion}

The feedback control performance is evaluated based on a weighted norm of the error between the reference and achieved values of the plasma parameters of interest. This performance measurement can also be used to compare the efficiency of this controller with other controllers that are using different control strategies and that are implemented in the same environment. The performance measures, that are used to measure the efficiency of the feedback control, are given by $J_{\beta}(t)$ to measure the performance of $\beta$ feedback control and $J_{\iota}(t)$ for the performance measurement of $\iota$.These functions are defined in [19] as:

$$
\begin{aligned}
J_{\beta}(t) & =\frac{(\beta(t)-\bar{\beta}(t))^{2}}{\bar{\beta}(t)^{2}} \\
J_{\iota}(t) & =\sum_{i=1}^{n_{p}} W\left(\rho_{i}\right) \frac{\left(\iota\left(x_{i}, t\right)-\bar{\iota}\left(\rho_{i}, t\right)\right)^{2}}{\bar{\iota}\left(\rho_{i}, t\right)^{2}}
\end{aligned}
$$

In other words, $J_{\iota}(t)$ is integral of weighted $\iota$ tracking error, where the spatial weighting $W(\rho)$ is defined in [19] and is used to highlight the part of the region of interest to measure the performance of the feedback control. In these control scenarios more emphasis is given to the region closer to the plasma centre. The total weighting norm for the performance measurement of the combined control of the two parameters is given by $J_{\text {tot }}(t)=v_{\beta} J_{\beta}(t)+v_{\iota} J_{\iota}(t)$, where the weights $v_{\beta}$ and $v_{\iota}$ are used to highlight the relative importance of the controlled plasma parameter of interest.

\section{Simulation results}

In the first phase, RAPTOR is run outside the TCV control environment as a simulator in stand-alone situation. The aim of this simulation environment is to have extensive tests of the feedback control performances before being applied in the real TCV experiments. Several control scenarios are considered to examine the robustness and performance of the controller. In these simulations the parameters of the RAPTOR 
simulator are chosen to be the same with the one of the experimental setup in TCV. The configuration of the reference values of the current and power distributions are the same as in the TCV experiments. In this configuration, the flat-top plasma current is set to a constant $I_{p}=120 \mathrm{kA}$, while the EC clusters are used as control inputs. One $P_{e c 1}$ (counter-ECCD) and one $P_{e c 2}$ (co-ECCD) clusters are used in the TCV experiments. In these RAPTOR simulations, $P_{e c 1}$ has an allowed power range of $100-750 \mathrm{~kW}$, and $P_{e c 2}$ has $360-900 \mathrm{~kW}$. The RAPTOR simulator solves the nonlinear coupled transport of $T_{e}$ and $\Psi$ represented by two coupled PDEs. The sawteeth effect is not included in this model. The $n_{e}$ profile is prescribed in the RAPTOR simulations, and in real experiments, it can be time-varying and provided by a profile reconstruction code. In the current version of the RAPTOR code, the temperature of the ions $T_{i}$ is estimated using pre-set ratio $f_{T i}(\rho)=T_{e} / T_{i}$ and $n_{i}$ is estimated using the assumption $n_{e} \approx n_{i}$.

The feedback control algorithm for the $\iota$ profile has available reconstructions obtained in $n_{p}=11$ equidistant $\rho$-grid points. To implement the control given by the optimization problem (35), we apply numerical integration of (36). The simplified model used to represent the ECCD current and power distributions permits the optimization problem (35) to be solved as a constrained linear least square problem. The extracted feedforward stationary values of the inputs that correspond to the desired reference profiles $\left(\bar{P}_{e c 1}, \bar{P}_{e c 2}\right.$ and $\left.\bar{I}_{p}\right)$ are calculated solving the static equation (9) and added to the feedback control. In RAPTOR simulations we can generate the target profiles when the corresponding feedforward input signals are applied sufficiently long to bring the plasma in a steady state. Using these references in the control simulations we can achieve a perfect tracking of the target profile in a steady state. However, this is not the case in most of the real experiments where it is difficult to achieve a perfect tracking of the target profile due to the limited degrees of freedom of the available actuators.

The PI feedback control of the $\beta$ parameter is calculated using a fixed values for the global energy confinement time $\tau_{t h}=4.4 \mathrm{~ms}$ and the possible effective time delay is taken also into consideration. The optimal value of the free parameter is fixed at $\tau_{c}=0.1$ after tuning, using simulation tests and choosing the best tracking performance. The $\iota$ controller parameter profiles, $\alpha_{p}(\rho)$ and $\alpha_{I}(\rho)$, that were used in these simulations are presented in Fig. 5a and Fig. 5b. The values of the control parameters were tuned such that they satisfy the stability criterion posed by Theorem 1. Because in these simulations we perform simultaneous control of the both parameters, the weighting functions are selected as $\omega_{1}=\omega_{2}=0.5$.

Fig. 4 presents the results from the closed-loop RAPTOR simulation where several different reference points are extracted and the control is tested to track these different points. In this simulations the control is activated at $t=0.1 \mathrm{~s}$. From the simulation results we can see that the closed-loop system successful tracks the multiple targets for both plasma parameters. In these plots we presented the performance of the control using different gains for the $\iota$ control, while the $\beta$ controller gain was the same for both simulations. The second control scenario (control scenario 2) has increased control gains $\alpha_{p}(\rho)$ and $\alpha_{I}(\rho)$ in comparison with the first control scenario (control scenario 1). The gains of the first control scenario 1 are given in Fig. 5a and the gains of the control scenario 2 are given in Fig. 5b. From these plots we can see the effect of these gains in the control performance. The features of these terms are very similar with the one used in the control of SISO systems. Increasing the value of the proportional gain increases the setting time and decreases the steady state error 

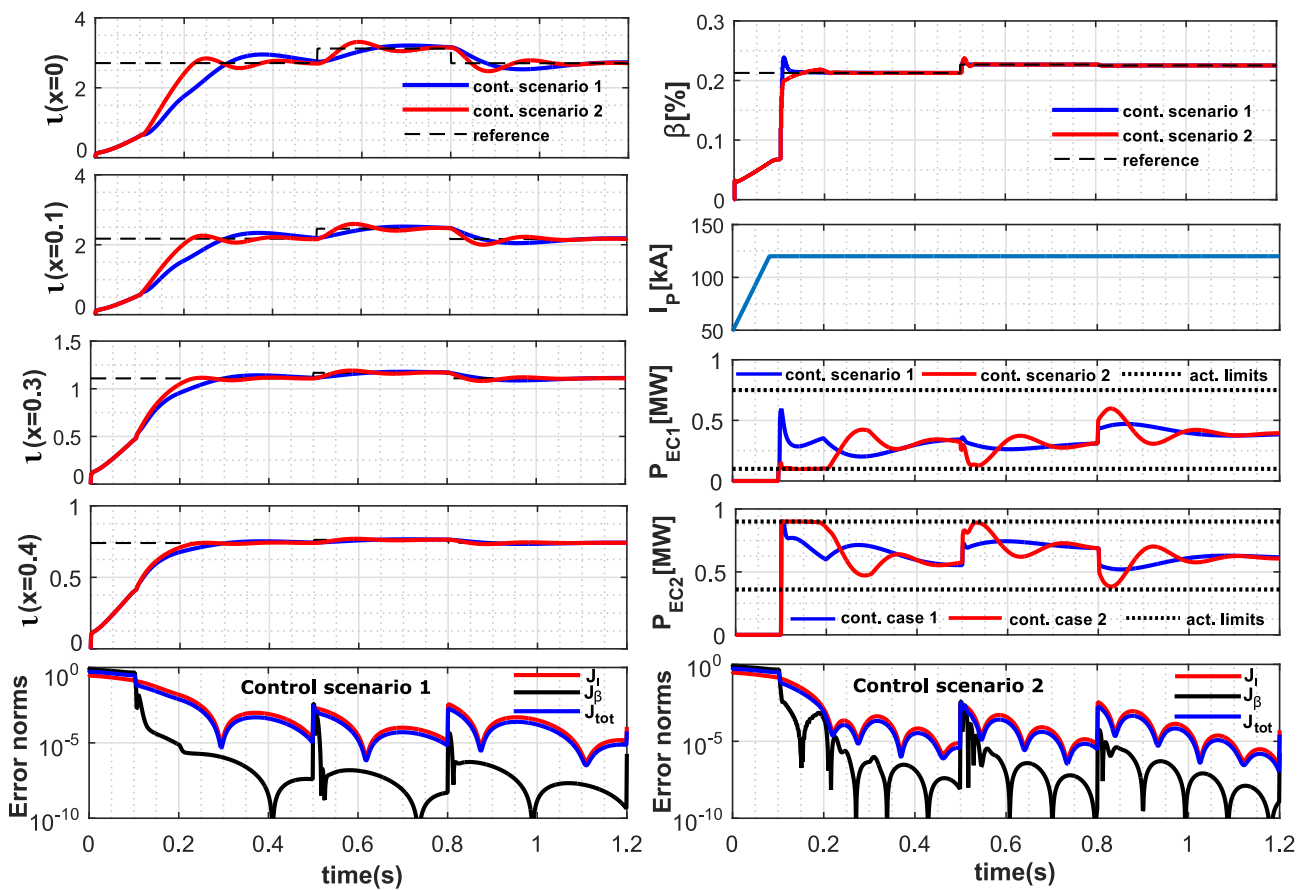

Figure 4: Evolution of $\iota, \beta, P_{\mathrm{aux}}, I_{\mathrm{p}}$ and the error norms in a closed-loop RAPTOR simulation.

while increasing the integral action eliminates the offset more efficiently. If these gains are too large, they can introduce oscillations and high sensitivity to high frequency disturbances.
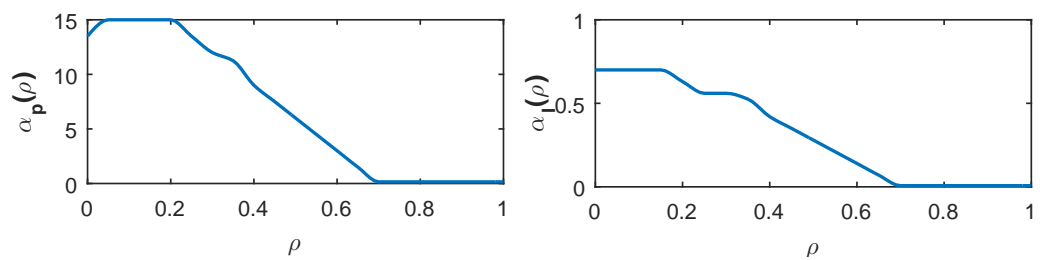

(a) $\iota$ controller parameters used in control scenario 1 .
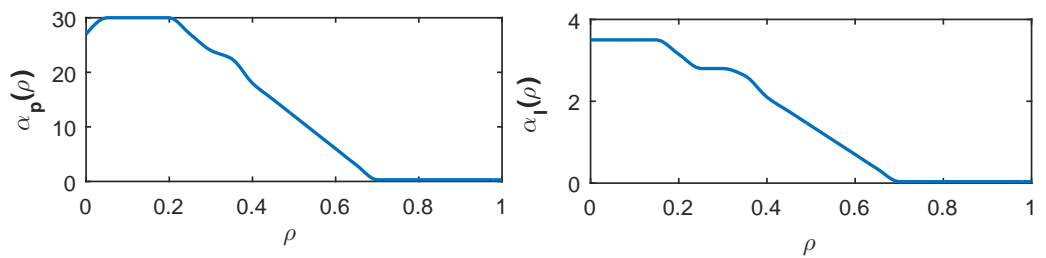

(b) $\iota$ controller parameters used in control scenario 2 .

Figure 5: Values of controller gains $\alpha_{p}$ and $\alpha_{I}$ used in the RAPTOR simulations.

In Fig. 6, the $\iota$ profile of the closed-loop system from another RAPTOR simu- 
lation is presented to illustrate the effect of using the parameter $\lambda(t)$. In the plots we are presenting two simulations using same parameters besides different $\lambda(t)$. In the first simulation the feedback control is activated and the forgetting factor $\lambda(t)$ is added to the integral action at $t=0.1 \mathrm{~s}$. In these RAPTOR simulations the components that define $\lambda(t)$ are set to $\kappa=0.08$ and $\sigma=10^{2}$. This parameter was active only during the transition period when the control was activated at $0.1 \mathrm{~s}$ and was set to zero afterwards to avoid steady state error. In the second simulation only pure integrator $(\lambda(t)=0)$ is used. In the transition intervals we can have an overshoot in the closed-loop tracking as a result of the integral action. From the plots we can conclude that by adding $\lambda(t)$ we improve the performance of the integral action by reducing these overshoots.
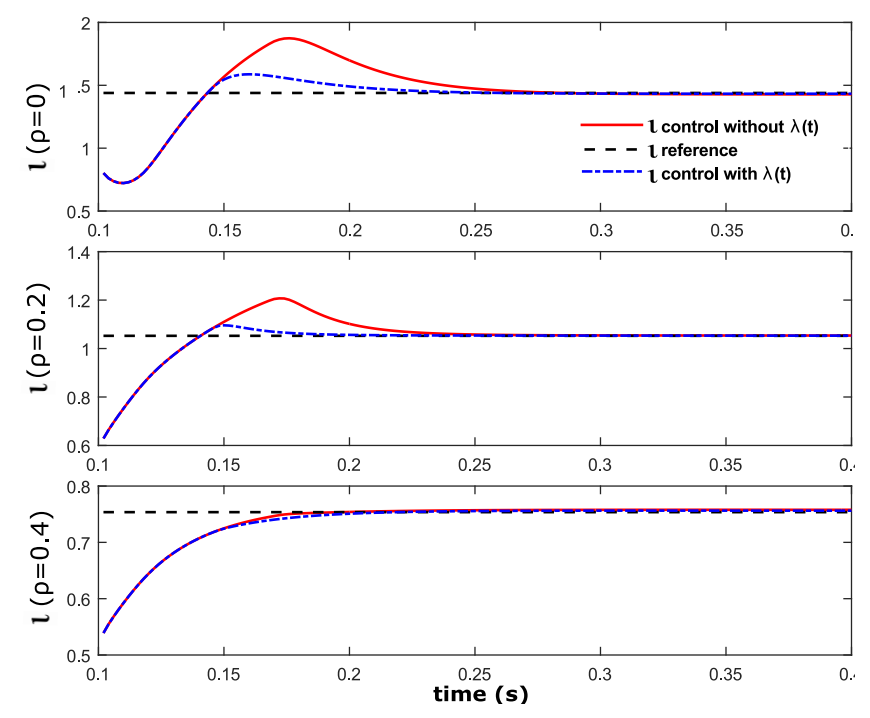

Figure 6: Response of $\iota$ with a pure integrator (red line) compared with the response when a forgetting factor $\lambda(t)$ is added to the integral action (blue dashed line) at several discrete locations.

\subsection{Controller performance with time delays}

Transportation of the control signals and their transition in the control systems can generate delays. Sometimes the effect of these delays cannot be neglected. For this reason, the performance of the control algorithm should be tested and adapted to these delays, which can be present when the control is applied on the real TCV control system. The control performance is tested by adding a $5 \mathrm{~ms}$ time delay in the plasma states from RAPTOR that enters in the controller, based on the possible time delays in the control environment.

The performance of the feedback control of the slow component $\iota$ are presented in Fig. 7. The time delays of small scales do not affect significantly the control of this component. In the case of a larger time delay of $100 \mathrm{~ms}$ and higher that can affect the performance, some extra tuning of the control parameters should be considered as discussed in [18]. The time delays in the system have more impact on the performance 

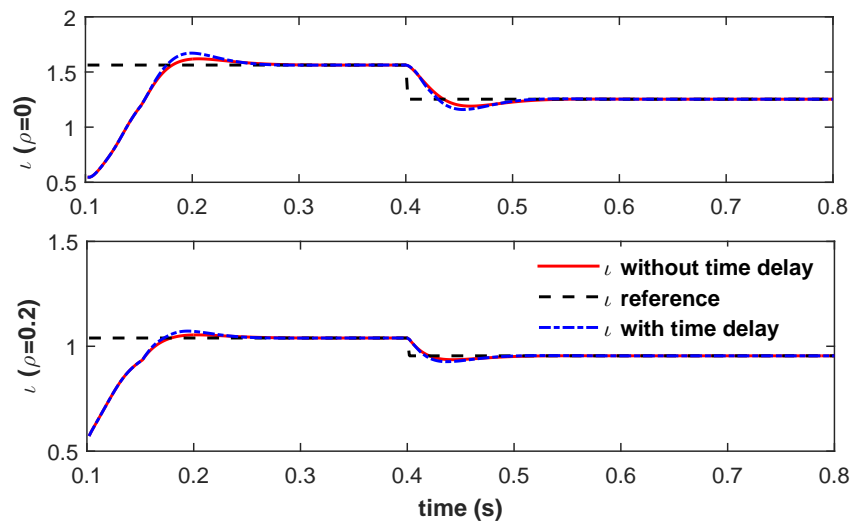

Figure 7: Tracking evolution of $\iota$ in the presence of a time delay in the feedback loop.

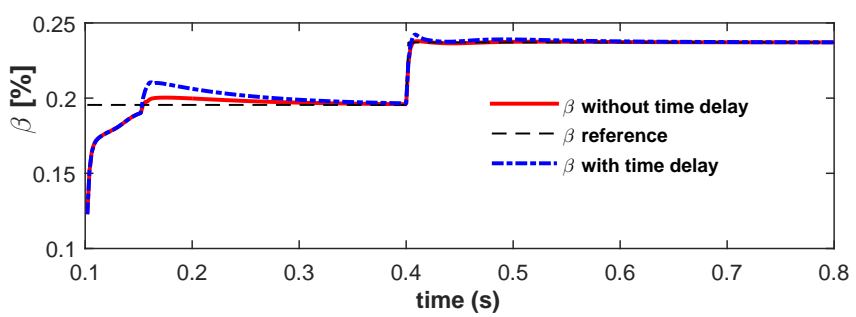

(a) $\beta$ tracking with $\tau_{c}=0.1$.

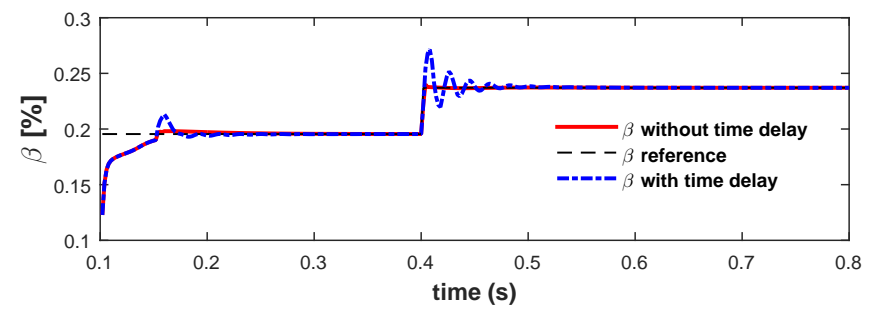

(b) $\beta$ tracking with $\tau_{c}=0.01$.

Figure 8: Tracking evolution of $\beta$ in a presence of a time delay of $5 \mathrm{~ms}$ in the feedback loop.

of the fast varying $\beta$ parameter than on $\iota$. The results of control for $\beta$ with $\tau_{c}=0.1$ and $\tau_{c}=0.01$ are presented in Fig. 8a and Fig. 8b, respectively. The robustness is increased and the convergence speed of the response is decreased by using an increased value of $\tau_{c}=0.1$. In this case the high overshoots and oscillations are effectively attenuated even when a time delay of $5 \mathrm{~ms}$ is present. In the case when $\tau_{c}=0.01$ is used the speed of the convergence is improved. On the other hand, when the time delay is introduced, there are higher overshoots and oscillations in the closed-loop tracking. 

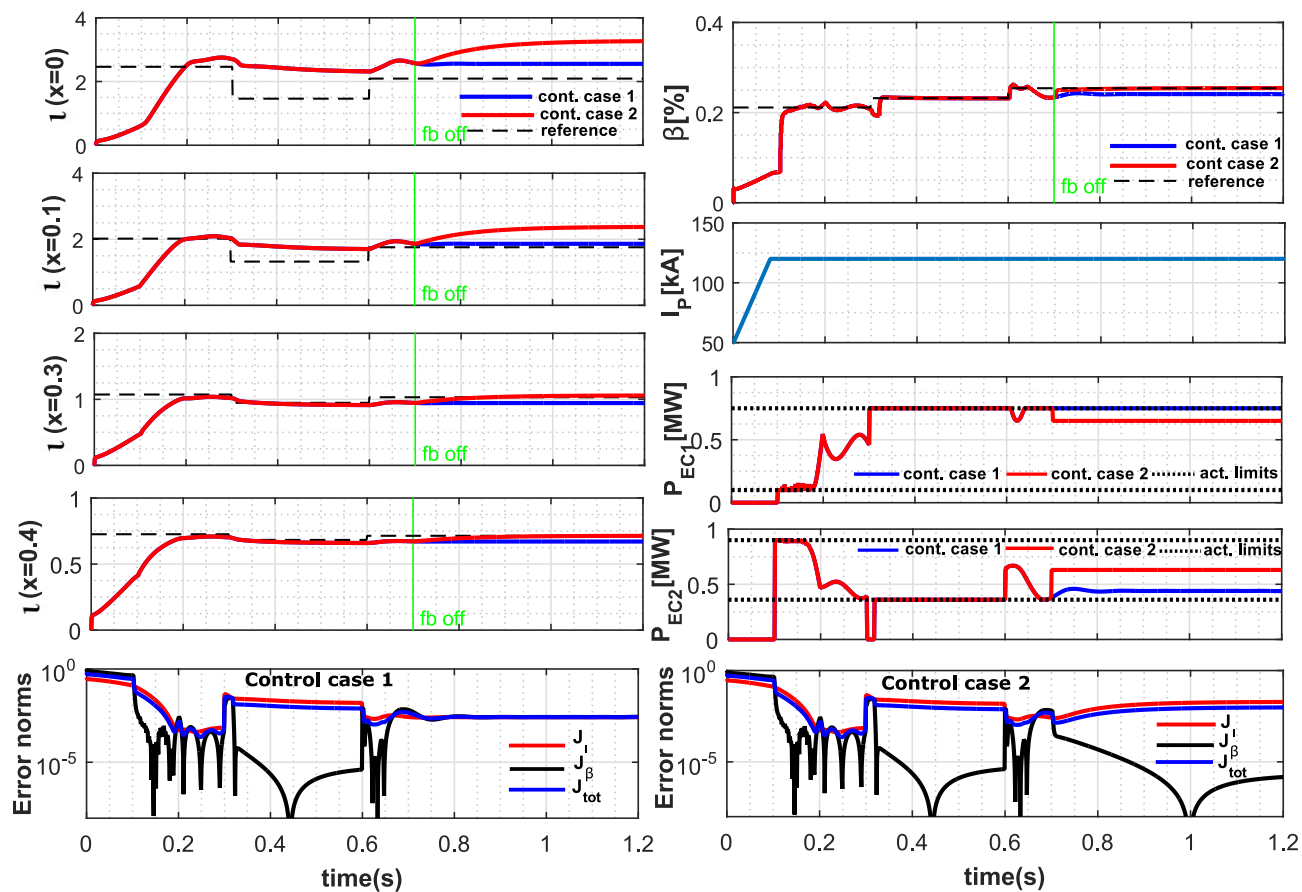

Figure 9: Evolution of $\iota, \beta, P_{\text {aux }}, I_{\mathrm{p}}$ and the error norms form the RAPTOR simulation with changed deposition location of the actuator $P_{e c 2}$. In control case 2 the feedback component is switched off at $t=0.7 \mathrm{~s}$ and only feedforward control is used.

\subsection{Change of the deposition location and width of $P_{e c 2}$}

The control performance is tested in a scenario where the deposition location and width of the ECCD clusters are different than the ones given by the nominal model. For this purpose, the deposition in the second ECCD cluster $\left(P_{e c 2}\right)$ is changed such that the width is increased by $12.5 \%\left(\omega_{d e p}=0.40\right)$ and the location of the peak of the deposition is $\rho_{\text {dep }}=0.05$. The desired reference profile is no longer reachable using the available inputs because of this perturbation that is introduced in the system. The simulation results for the tracking of $\iota$ profile and $\beta$ are presented in Fig. 9. In these simulations we considered two control cases. In the first scenario the control is activated at $t=0.1 \mathrm{~s}$ (control case 1) and the control is active until the end of the simulation. While in the second scenario (control case 2) the feedback control was activated in the time window $t=0.1-0.7 \mathrm{~s}$. The feedback control is deactivated after $0.7 \mathrm{~s}$ to compare the performance of the feedback control with the case where only the feedforward inputs are used. From Fig. 9, it can be clearly concluded that the feedback control improves the control performance of the $\iota$ profile by reducing the offset. In both cases a good tracking of $\beta$ is still achieved because this parameter depends less on the deposition location and width of the ECCD clusters. Also the results reveal that $q_{0}$ is very sensitive to actuator settings. 

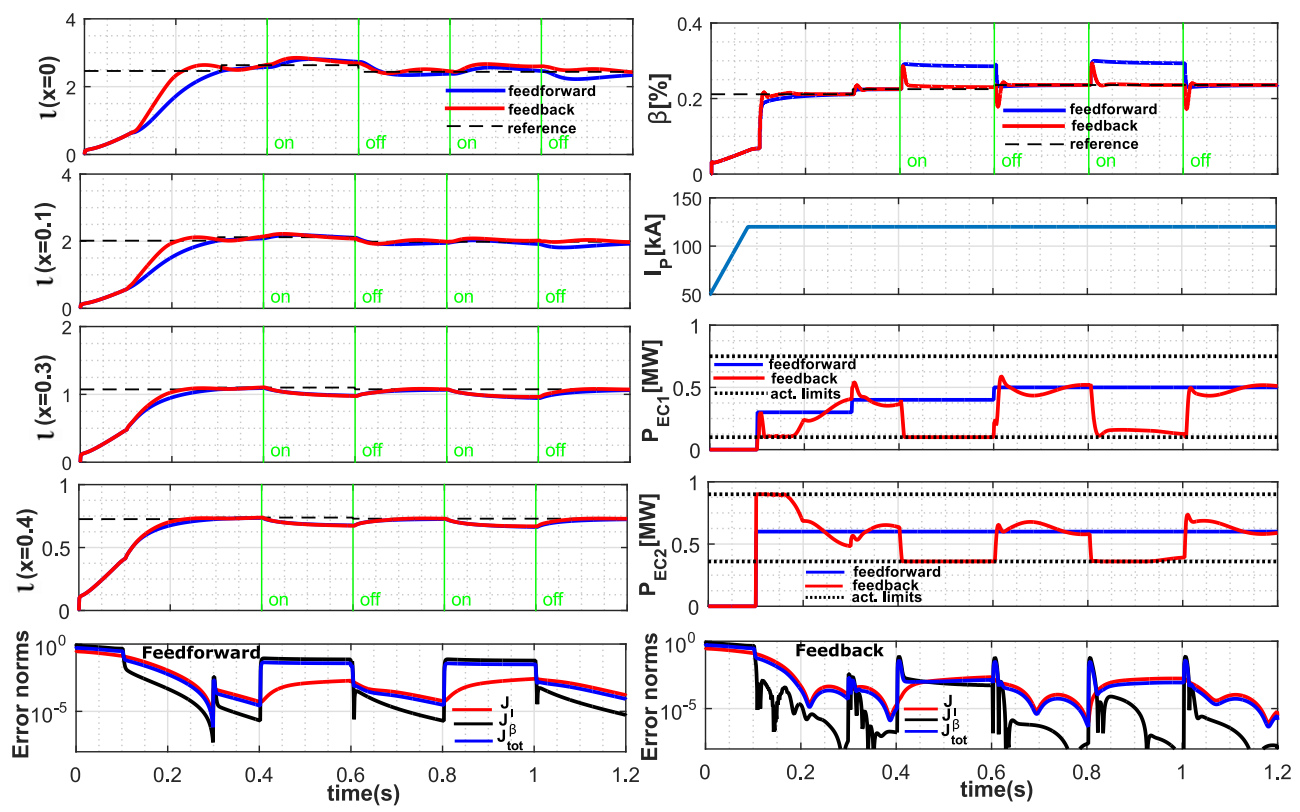

Figure 10: Evolution of $\iota, \beta, P_{\text {aux }}, I_{\mathrm{p}}$ and the error norms from RAPTOR simulation with added NBI heating as a source of disturbance. The input power is added at the time intervals $t \in[0.4,0.6] \mathrm{s}$ and $t \in[0.8,1] \mathrm{s}$ with a power of $1 \mathrm{MW}$.
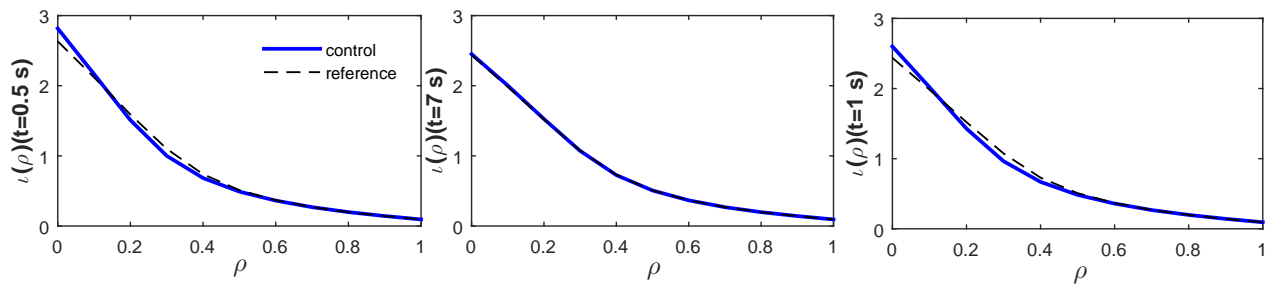

Figure 11: Profile of $\iota$ at $t=0.5 \mathrm{~s}, 0.7 \mathrm{~s}$ and $1 \mathrm{~s}$ from the RAPTOR simulation with added NBI heating as a source of disturbance.

\subsection{Disturbance attenuation}

The sensitivity of the control algorithm to disturbances and their rejection is one of the most important features that should be tested before the controller is applied. In a complex system like the tokamak, where the model of the system depends on numerous parameters, there can exist many of sources of disturbances. To test the disturbance attenuation of the controller, an additional Neutral Beam Injection (NBI) with power and co-current drive was added to the simulations to act as a source of external disturbances. The model for the NBI that is used in RAPTOR simulator is simple and uses Gaussian distribution for the current density and for the heating power density. However, the accuracy of the model for these simulations is less important, since the main goal of this NBI is to act only as a disturbance source. The control of the system is activated at $t=0.1 \mathrm{~s}$ and the disturbing source was activated during 
the time intervals $t \in[0.4,0.6] \mathrm{s}$ and $t \in[0.8,1] \mathrm{s}$ with a power of $1 \mathrm{MW}$. The results form the RAPTOR simulation are presented in Fig. 10. When the NBI heating is added the plasma parameters deviate from the target values and the feedback control algorithm tends to bring the plasma parameters to the desired references. In the plots we included the results from two simulations. In the first case only the feedfoward inputs were used, while in the second case the feedback controls was added to the feedfoward signals. From the results we can see that the feedback action successfully manages to attenuate the disturbance that comes from the NBI source. We can also confirm that the feedback control accelerates the convergence rate to the target state. Resulting $\iota$-profile for this simulation at several time instants is presented in Fig. 11.

\section{TCV experiments}

After the extensive tests of the control algorithm using the RAPTOR simulator, the control algorithm is transferred and implemented in the TCV control system. The control environment [19] permits the MATLAB software for the controller to be used directly in the experiments.

The RAPTOR code is incorporated as an observer in the TCV control environment, which provides the essential real-time estimates of the $T_{e}$ and $\Psi$ profiles in several $\rho$-grid points. The $T_{e}$ and $\Psi$ profiles are reconstructed by the RAPTORobserver using an extended Kalman filter scheme [21, 44]. These profile estimates

are necessary to calculate the estimates of $\iota \propto \frac{1}{\rho} \frac{\partial \Psi}{\partial \rho}$ profile and $\beta \propto \int_{0}^{1} V^{\prime} n_{e} T_{e} d \rho$ for our control purposes. The TCV diagnostic system lacks measurements of the internal magnetic field in the plasma region. Thus, the estimated $\iota$ profile is calculated using only the RAPTOR model predictions constrained by the plasma geometry and experimental total plasma current measurement. The effect of sawteeth was not considered in the model, which results to physically unrealistic estimate of $\iota$ profile that is much larger than 1 in many cases. However, this drawback does not affect the test of the performance of the presented control algorithm because the estimated and the reference profiles were generated using the same model. More details about the accuracy of the estimates and comparison of the profiles with off-line profile reconstructions using LIQUE [45] and ASTRA [25] can be find in the paper dedicated to the description of the control environment [19].

The volume-averaged density in the TCV experiments is calculated in real-time using the code described in [46]. The electron density is controlled using robust density controller [47] and this control is executed in parallel with the profile control in the TCV control system. The density is chosen low $\left(n_{e}(\rho=0)=2 \cdot 10^{19} \mathrm{~m}^{5}\right)$ in order to maximize the ECCD current. See [19, 48, 49] for more details on the TCV control system.

In the TCV experiments the plasma current $I_{p}$ is controlled by the inductive voltage from external coils, and can be adjusted in real-time. The value of this parameter is set to the desired value and is not included in our feedback control synthesis. In these experiments the cluster $P_{e c 1}$ has time varying power limits. The range of this cluster is $100-750 \mathrm{~kW}$, with an extra restriction that the minimal allowed power increases to $550 \mathrm{~kW}$ if $P_{e c 1}<500 \mathrm{~kW}$ for more than $0.47 \mathrm{~s}$. The power of cluster $P_{e c 2}$ is in the range of $360-900 \mathrm{~kW}$.

RAPTOR observer provides the estimate of the $\iota$ profile at 11 equidistant $\rho$-grid points in the range of $\rho \in[0: 1]$ for the control purposes. For the control synthesis a linear model was used that was extracted by applying static inputs $I_{p}=120 \mathrm{kA}$ 
and $P_{e c 1}=P_{e c 1}=500 \mathrm{~kW}$. The feedforward signal of the control is calculated in real time using the lumped representation of the linearized model of (9) at several $\rho$-grid points. The corresponding signals were calculated as variations around this model. The small offset that can appear as a result of the nonlinear nature of the system is compensated easily by the integral action of the control. In the TCV experiments the optimization problem (35) is solved as a linear least square problem, without including the limits of the actuators. This permits easy implementation using only matrix multiplication and inversion, without significantly affecting the performance. The anti-windup compensator that is implemented in the TCV control system is updated with the real time actuator limits which are crucial for its performance. The simplicity of the control algorithm presented in this paper allows it to be executed in a short time and easily applicable within the TCV control system.
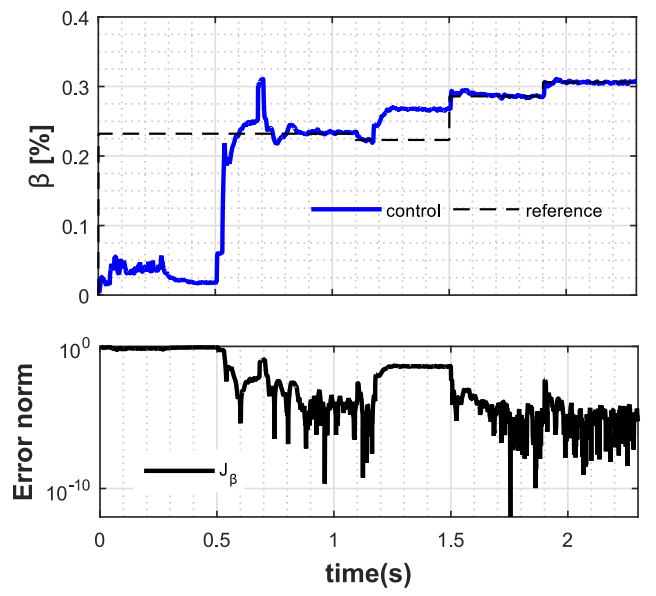

TCV \#54839
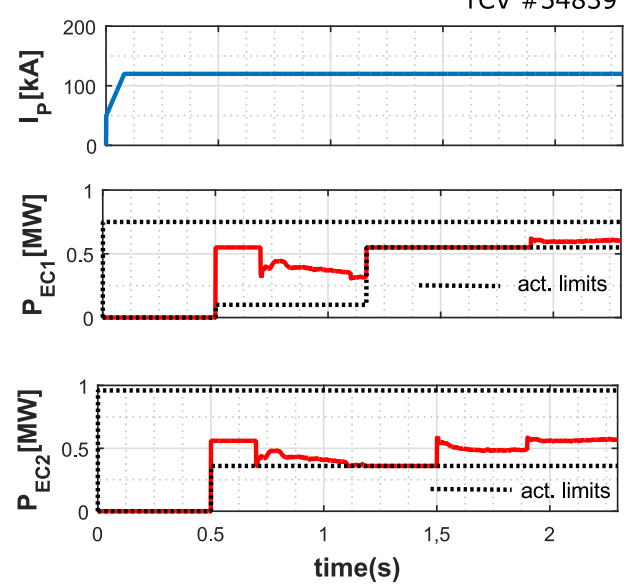

Figure 12: Evolution of $\beta, P_{\operatorname{aux}}, I_{\mathrm{p}}$ and the error norm in shot \# 54839. Two ECCD clusters are used as control inputs and the value of $I_{p}$ is fixed.

The control strategy is tested in several TCV shots where different sets of control parameters are used. In the shot \# 54839 only control of the pressure parameter $\beta$ is considered. In these experiments the same $\beta$ controller gains were used as in the simulations. In this experiment the controller should track several targets for $\beta$. The results from this shot are presented in Fig. 12. From the RAPTOR observer outputs we can see that the $\beta$ controller successfully tracks the different targets. In the case where only $\beta$ control is active, the total power is equally shared between the two clusters, thus the powers delivered by the two inputs are equal until one of the clusters saturates. Because of the variable power limitations of the first cluster $P_{e c 1}$, after around $1.2 \mathrm{~s}$ the minimum limit of this actuator rises to $550 \mathrm{~kW}$ and the target state is no longer reachable. At $1.5 \mathrm{~s}$ and $2 \mathrm{~s}$ the target states were increased and they were successfully reached by the feedback controller.

The control of the $\iota$ profile is applied in shot \# 54838. The values of the controller parameters $\alpha_{p}(\rho)$ and $\alpha_{I}(\rho)$ used in this shot are presented in Fig. 15a, and $\lambda(t)=0$ was used. Since we have only two degrees of freedom given by the power delivered by the two actuators and several controlled points of the $\iota$ profile, the target profile should be chosen a priori such that it can be achieved with a limited number of actuators. From the plots shown in Fig. 13 we can see that the feedback control manages to reach 

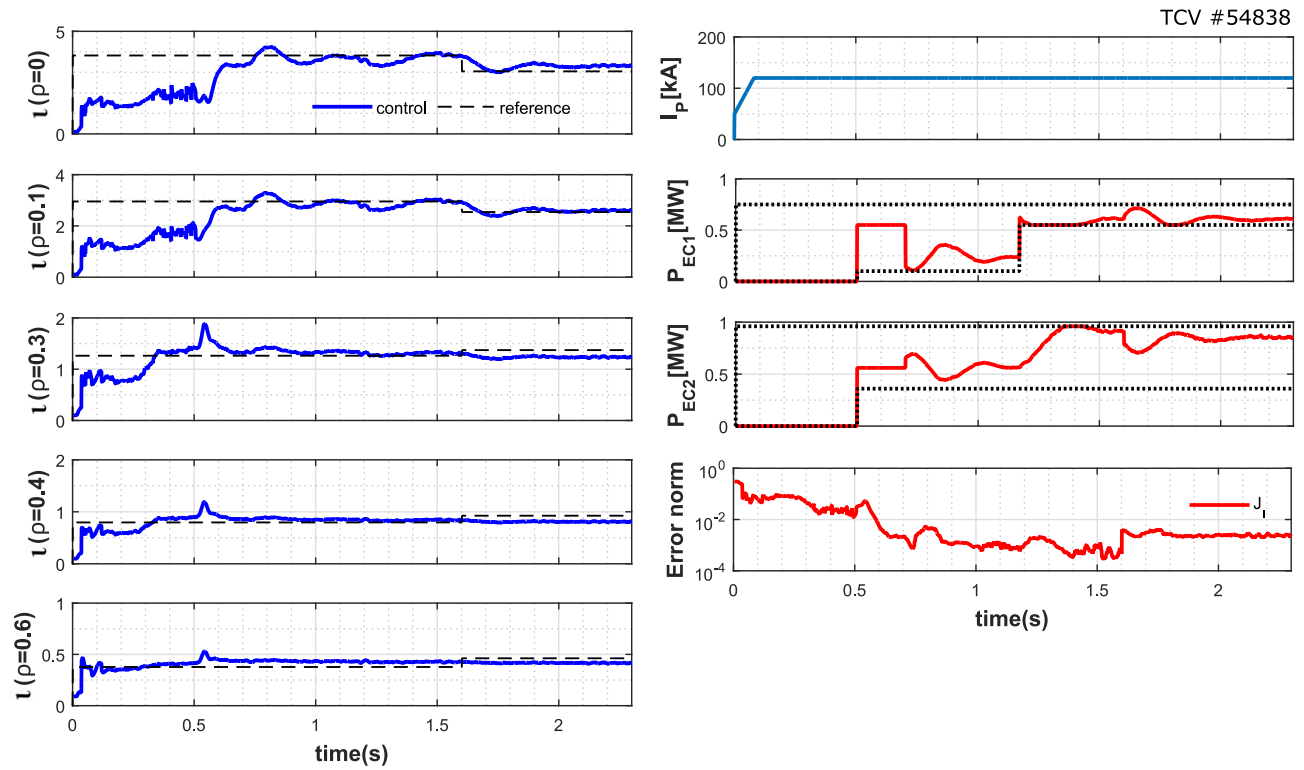

Figure 13: Evolution of $\iota, P_{\text {aux }}, I_{\mathrm{p}}$ and the error norm in shot \# 54838. Two ECCD clusters are used as control inputs and the value of $I_{p}$ is fixed.
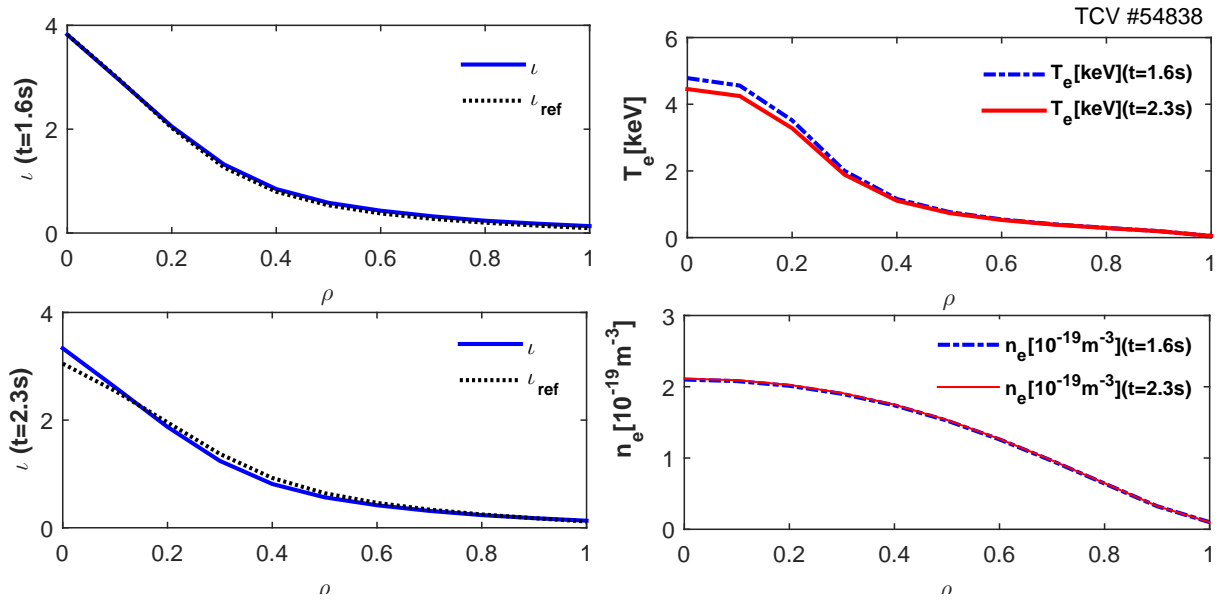

Figure 14: Profiles of $\iota, T_{e}$ and $n_{e}$ in shot \# 54838.

the desired target state after the activation of the control. In the $\iota$ profile plots we can observe certain oscillations that are coming from the increased integral action and also from the model uncertainties that are coming from different plasma phenomena. To reduce these oscillations in future experiments, the controller gains should be reduced and also techniques to filter the uncertainties can be applied. Because of the change of the minimum allowed power for the first cluster, at around $1.2 \mathrm{~s}$ the power $P_{e c 1}$ increases to $550 \mathrm{~kW}$ which results in an increase of $P_{e c 2}$ to compensate this change. At $1.6 \mathrm{~s}$ the target $\iota$ profile is changed and the feedback control aims at the new desired profile. The resulting and the target $\iota$ profile at $t=1.6 \mathrm{~s}$ and $t=2.3 \mathrm{~s}$ and 
the temperature profile are presented in Fig. 14. From the plots we can see that not all the desired target states were reachable. This is because the reference parameters for the second target were generated by using high $I_{p}=150 \mathrm{kA}$. The feedback control applied using only the EC clusters gives more emphasis on the desired profile states closer to the center of the plasma. To achieve a better tracking of the points that are closer to the plasma edge, it is necessary to include also $I_{p}$ as an boundary actuator.
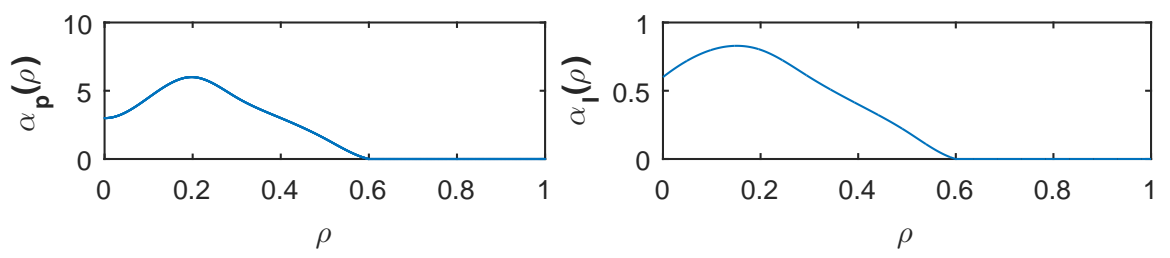

(a) $\iota$ controller gains used in the TCV shot \#54838.
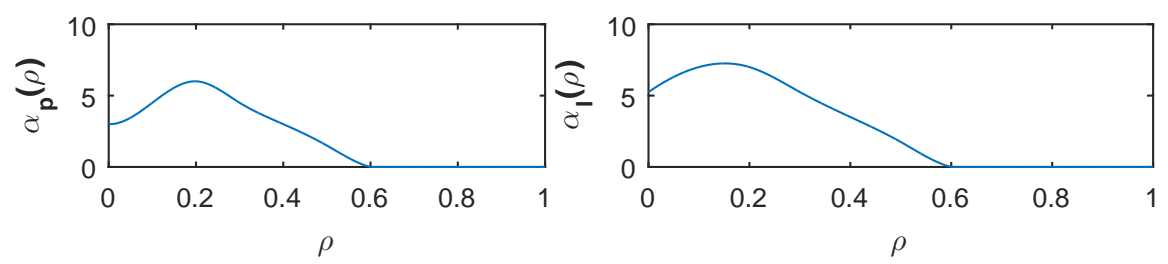

(b) $\iota$ controller gains used in the TCV shot \#54835.

Figure 15: Values of controller gains $\alpha_{p}$ and $\alpha_{I}$ used in the TCV shots.

Simultaneous control of both $\iota$ and $\beta$ is performed in shot \# 54835. The values of the controller parameters $\alpha_{p}(\rho)$ and $\alpha_{I}(\rho)$ used in this shot are presented in Fig. 15b, and $\lambda(t)=0$ was used. The results from this shot are presented in Fig. 16. The resulting and the target $\iota$ profile at $t=1.6 \mathrm{~s}$ and $t=2.3 \mathrm{~s}$ and the temperature profile are presented in Fig. 17. The control algorithm manages to achieve the desired $\iota$ profile and $\beta$ targets at the beginning of the control. When the targets for both plasma quantities are changed at $1.6 \mathrm{~s}$, these targets cannot be reached and the actuators are in saturation. As in the case of shot \# 54838, the second target at $1.6 \mathrm{~s}$ were generated using $I_{p}=150 \mathrm{kA}$ and they were not reachable using fixed $I_{p}=120 \mathrm{kA}$ but are nevertheless relatively well reproduced within the actuator limits.

\section{Conclusions and future work}

In this paper we presented feedback control algorithms for simultaneous control of the plasma $\iota$ profile and the $\beta$ pressure quantity. The control algorithms were successfully tested using simulations and real TCV experiments.

The results show a successful simultaneous tracking of $\iota$ and $\beta$ using two EC clusters. The performance of the control using several control scenarios in presence of different uncertainties and disturbances was tested in RAPTOR simulations prior to the experiments. Finally, the feedback control algorithms were implemented on the TCV control system and tested in several shots.

While our algorithm is easy to be adopted to other in-domain actuators (auxiliary heating and current drive), it requires modifications or combination with other control methods to be extended to include boundary actuators. A boundary control algorithm 

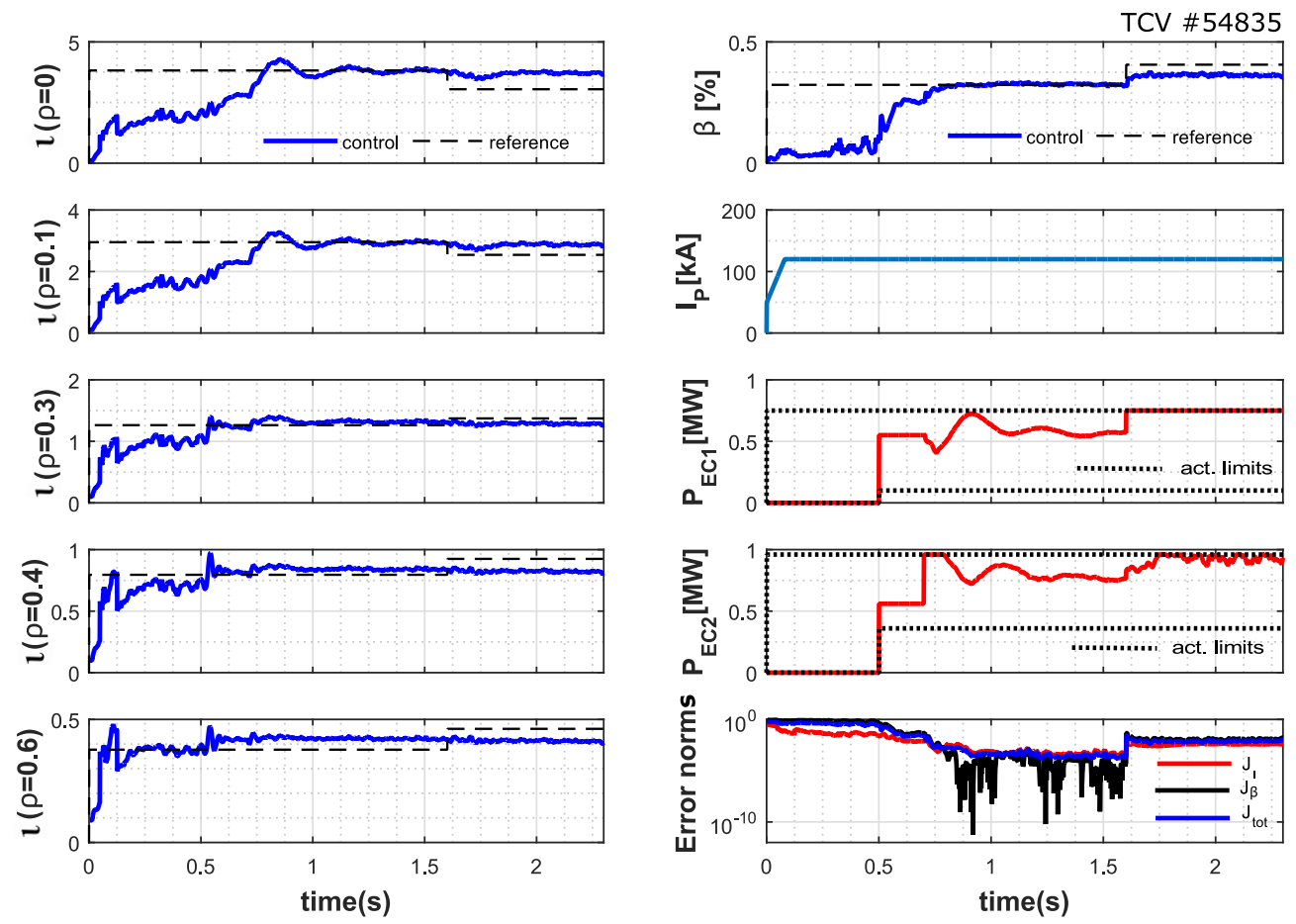

Figure 16: Evolution of $\iota, \beta, P_{\mathrm{EC}}, I_{\mathrm{p}}$ and the error norms in shot in shot \# 54835 . Two ECCD clusters are used as control inputs and the value of $I_{p}$ is fixed.
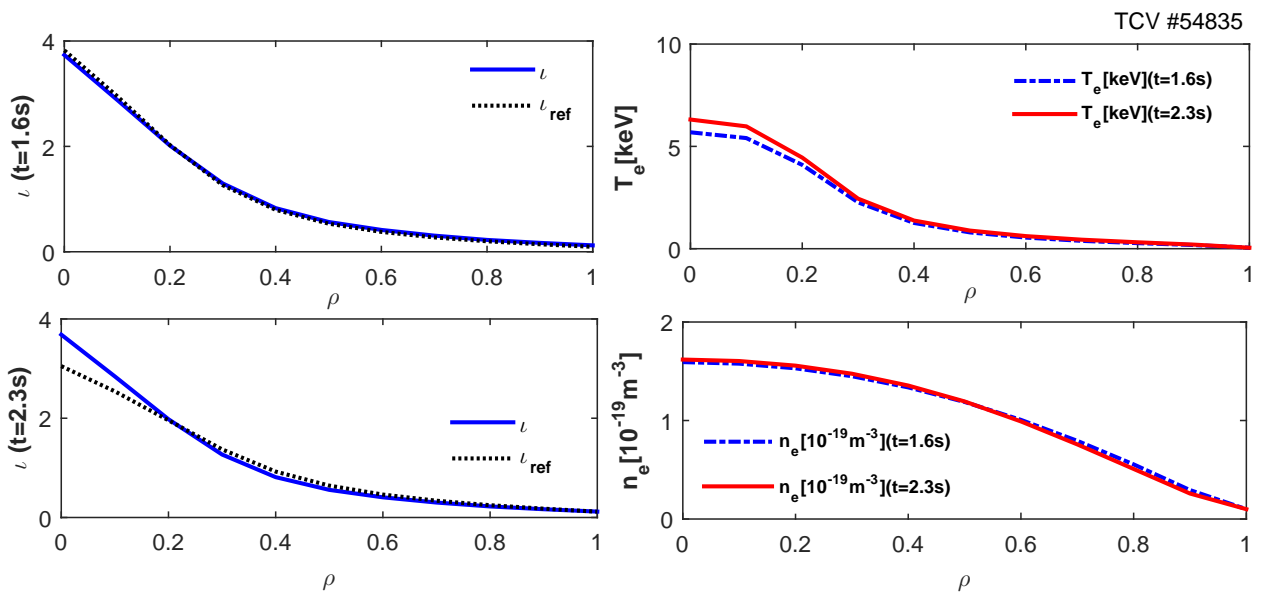

Figure 17: Profiles of $\iota, T_{e}$ and $n_{e}$ in shot \# 54835. 
applied to PDEs, that includes the boundary input $I_{p}$ controlled directly by the loop voltage, could eventually improve the performance of the system $[50,51]$. Direct application of these infinite-dimensional boundary control methods is difficult as a result of the complexity of the equation. However, a spatially discretized version of the backstepping approach that includes $I_{p}$ as a control input is already applied for the control of the current profile and experimentally tested on DIII-D in L-mode [7] and in H-mode [52]. In [52] the boundary control method is extended to include in-domain control.

The unified framework for different control strategies if this TCV experimental campaign permits us to compare the performance of the different controllers that were implemented. One of the most successful control performances are done by using Model Predictive Control (MPC). MPC algorithms for the control of the plasma safety factor are developed in several works $[19,53,54]$. These algorithms are more complex and require implementation of quadratic programming solver that demanded bigger computational power. Successful testing of the MPC in this experimental campaign [19] shows very good handling of the actuator constraints that can be included explicitly in the MPC synthesis. A disturbance observer to estimate the slowly varying component is used to reduce the steady state error in this controller. This is equivalent to the objective of the integral action that is applied in our design. The disturbance observer gives a good transition between different target states and good tracking performance with reduced overshoots. Some of the techniques applied in the MPC controller can be used as an insight to be adopted to this control algorithm to improve the control performance. Qualitative comparison of the implemented MPC with the robust control $[11,13]$ and the IDA-PBC [5] can be found in [55].

The main advantages of the proposed algorithm in this paper is its simplicity to be implemented the TCV control system and the fast computational time. It can also incorporated easily some techniques (i.e anti-windup, proportional-integral term) that can improve the control performance. With the proposed Lyapunov approach we have stability margin that gives us some robustness to the uncertainties that can appear in the control design and implementation. The main drawbacks of this algorithm is that it has several parameters that must be tuned before it can be applied in real experiments. This requires very good simulator that reflects accurate the dynamics of the system. Moreover, the infinite-dimensional approache demands estimation of the plasma profiles in many grid points in order to be implemented, that is not always the case. In future works this algorithm should also be extended to include the dynamics and the terms that were neglected in our simplified control model, but that could influence the performance in other plasma operation mode.

\section{Acknowledgments}

This work has been carried out within the framework of the EUROfusion Consortium and has received funding from the Euratom research and training programme 20142018 under grant agreement No 633053. The views and opinions expressed herein do not necessarily reflect those of the European Commission. This work is also supported by the ANR project TORID contract number ANR-12-BS03-0008, Swiss National Science Foundation and the Netherlands Organisation for Scientific Research (NWO) via the Innovational Research Incentives Scheme. 


\section{Appendix A. Proof of Theorem 1}

Proof. The time-derivative of the Lyapunov function is:

$$
\dot{\mathcal{V}}=\dot{\mathcal{V}}_{z}+\dot{\mathcal{V}}_{I}
$$

where

$$
\begin{aligned}
\dot{\mathcal{V}}_{z}(t)= & \int_{0}^{1} \rho^{2} p_{z}(\rho) \frac{\partial \tilde{z}}{\partial t} \tilde{z} d \rho \\
= & \int_{0}^{1} \rho^{2} p_{z}(\rho) \frac{\partial}{\partial \rho}\left(\frac{a_{1}(\rho)}{\rho} \frac{\partial}{\partial \rho}\left(a_{2}(\rho) \tilde{z}\right)\right) \tilde{z} d \rho \\
& -\int_{0}^{1} \rho^{2} p_{z}(\rho) \alpha_{p}(\rho) \tilde{z}^{2} d \rho+\int_{0}^{1} \rho^{2} p_{z}(\rho) \tilde{z} \mathcal{I}_{\iota} d \rho
\end{aligned}
$$

Applying integration by parts in $\dot{\mathcal{V}}_{z}(t)$ and defining $k_{z}(\rho)=\alpha_{p}(\rho) p_{z}(\rho)$ we get:

$$
\begin{aligned}
\dot{\mathcal{V}}_{z}(t)= & \left.\rho p_{z}(\rho) \tilde{z} a_{1}(\rho) \frac{\partial}{\partial \rho}\left(a_{2}(\rho) \tilde{z}\right)\right|_{0} ^{1}-\int_{0}^{1} \rho p_{z}(\rho) a_{1}(\rho) a_{2}(\rho)\left(\frac{\partial \tilde{z}}{\partial \rho}\right)^{2} d \rho \\
& -\int_{0}^{1}\left(2 p_{z}(\rho)+\rho p_{z}^{\prime}(\rho)\right) a_{1}(\rho) \frac{\partial a_{2}(\rho)}{\partial \rho} \tilde{z}^{2} d \rho \\
& -\int_{0}^{1}\left(2 p_{z}(\rho) a_{2}(\rho)+\rho p_{z}(\rho) \frac{\partial a_{2}(\rho)}{\partial \rho}+\rho p_{z}^{\prime}(\rho) a_{2}(\rho)\right) a_{1}(\rho) \tilde{z} \frac{\partial \tilde{z}}{\partial \rho} d \rho \\
& -\int_{0}^{1} \rho^{2} k_{z}(\rho) \tilde{z}^{2} d \rho+\int_{0}^{1} \rho^{2} p_{z}(\rho) \tilde{z} \mathcal{I}_{\iota} d \rho
\end{aligned}
$$

Calculating the time derivative of $\dot{\mathcal{V}}_{I}=\int_{0}^{1} \rho^{2} p_{I}(\rho) \frac{\partial \mathcal{I}_{\iota}}{\partial t} \mathcal{I}_{\iota} d \rho$ and defining $k_{I}(\rho)=$ $\alpha_{I}(\rho) p_{I}(\rho)$, we get:

$$
\dot{\mathcal{V}}_{I}(t)=-\int_{0}^{1} \rho^{2} k_{I}(\rho) \tilde{z} \mathcal{I}_{\iota} d \rho-\int_{0}^{1} \rho^{2} p_{I}(\rho) \lambda(t) \mathcal{I}_{\iota}^{2} d \rho
$$

Combining the equations $(\mathrm{A} .3)-(\mathrm{A} .4), \dot{\mathcal{V}}$ can be presented as:

$$
\dot{\mathcal{V}}(t)=\int_{0}^{1}\left[\begin{array}{c}
\tilde{z} \\
\frac{\partial \tilde{z}}{\partial \rho} \\
\mathcal{I}_{\iota}
\end{array}\right]^{T}\left[\begin{array}{ccc}
c_{1,1}(\rho) & c_{1,2}(\rho) & c_{1,3}(\rho) \\
0 & c_{2,2}(\rho) & 0 \\
c_{1,3}(\rho) & 0 & c_{3,3}(\rho)
\end{array}\right]\left[\begin{array}{c}
\tilde{z} \\
\frac{\partial \tilde{z}}{\partial \rho} \\
\mathcal{I}_{\iota}
\end{array}\right]
$$

where $c_{1,1}, c_{2,2}, c_{1,2}, c_{1,3}, c_{3,3}$ are defined in the statement of Theorem 1 . Considering $(25)$, we get $\dot{\mathcal{V}} \leq-\frac{1}{2} \gamma_{1} \int_{0}^{1} \rho^{2} p_{z}(\rho) \tilde{z}^{2} d \rho-\frac{1}{2} \gamma_{2}(t) \int_{0}^{1} \rho^{2} p_{I}(\rho) \mathcal{I}_{\iota}^{2} d \rho$. This concludes the proof of Theorem 1.

\section{Appendix B. Computation of the Lyapunov function}

The exponential stability of the system is verified by solving inequality (25) in Theorem 1. The solution of this inequality is found by transforming the inequality in 
to an Linear Matrix Inequality (LMI) problem by representing the weights of the Lyapunov functions, $p_{z}(\rho), p_{I}(\rho)$ and the control gains $k_{z}(\rho), k_{I}(\rho)$ as Legendre polynomials. Legendre polynomials are orthogonal in the range $[-1,1]$, and the polynomials $p_{z}(\rho), p_{I}(\rho), k_{z}(\rho)$ and $k_{I}(\rho)$ may be expanded in this interval in terms of them as [56]. The $p_{z}(\rho)$ can be presented as:

$$
p_{z}(\rho)=\sum_{i=0}^{N_{1}} c_{1, i} P_{i}(\rho)
$$

where $c_{1,1}, \ldots, c_{1, N_{1}}$ are constant that need to be found, $P_{i}(x)$ is $i-t h$ order Legendre polynomial and $N_{1}$ is the order of the Legendre polynomial for $p_{z}$. The same representation is applied for $p_{I}(\rho), k_{z}(\rho)$ and $k_{I}(\rho)$.

Sampling the interval $[0,1]$ and representing $p_{z}(\rho), p_{I}(\rho), k_{z}(\rho)$ and $k_{I}(\rho)$ as a sum of Legendre polynomials permits us to formulate the following LMI problem:

For given parameters $\gamma_{1}$ and $\gamma_{2}$, find $p_{z}(\rho), p_{I}(\rho), k_{z}(\rho)$ and $k_{I}(\rho)$ such that:

- $A(\rho)+A_{1}(\rho) \leq 0, \forall \rho \in[0,1]$;

- $\alpha_{l} W_{p}(\rho)<p_{z}(\rho) \leq \alpha_{h} W_{p}(\rho)$ and $\alpha_{l} W_{p}(\rho)<p_{I}(\rho) \leq \alpha_{h} W_{p}(\rho), \forall \rho \in[0,1]$;

- $k_{z}(\rho)>0$ and $k_{I}(\rho)>0$

- $\eta_{\|}(\rho)=\omega_{\eta} \eta_{\|, \min }(\rho)+\left(1-\omega_{\eta}\right) \eta_{\|, \max }(\rho), \forall \omega_{\eta} \in[0,1]$.

The LMI is solved to guarantee the stability of the closed-loop system for different values of $\lambda_{\max } \geq \lambda(t)>0$. The LMI solver YALMIP [57] is used to find the unknown constant polynomial coefficients in $p_{z}(\rho), p_{I}(\rho), k_{z}(\rho)$ and $k_{I}(\rho)$.

The shape of the weighting polynomials of the Lyapunov function $p_{z}(\rho)$ and $p_{I}(\rho)$ are constrained by the lower limit curve $\alpha_{l} W_{p}(\rho)>0$ and the upper limit curve $\alpha_{h} W_{p}(\rho)>0$, where the weigthing function $W_{p}(\rho)$ is chosen such that it highlights the area of $\rho$ which is the most important for the control performance. In these experiments the shape of $W_{p}(\rho)$ is chosen according to the weighting function $W(\rho)$ defined in the performance criteria (37). The plots of the solutions of the polynomials $p_{z}$ and $p_{i}$ in a case where these constraints are applied are presented in Fig. B1.
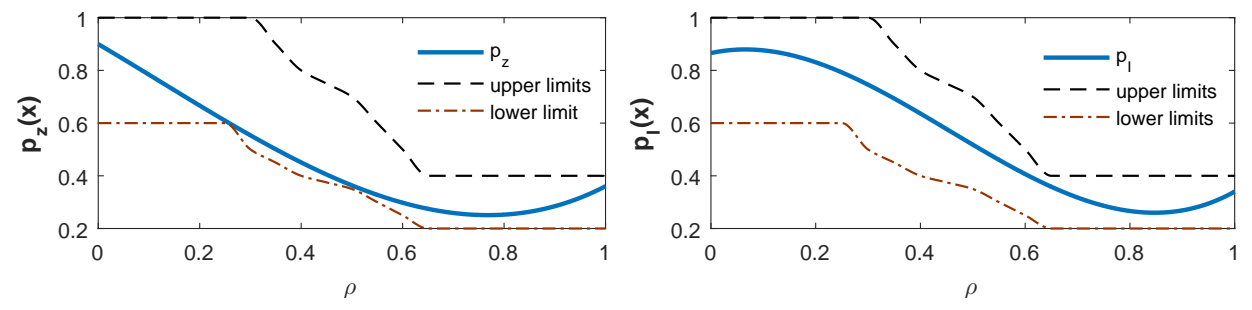

Figure B1: Plots of the solution $p_{z}$ and $p_{I}$ and the constrained. 


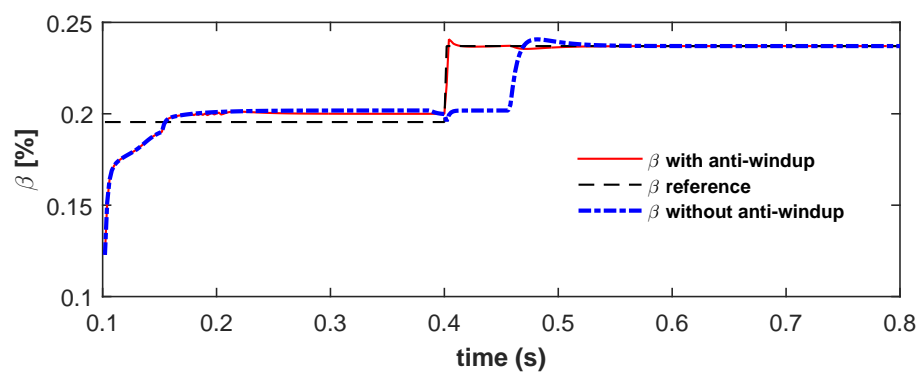

Figure C1: RAPTOR simulations comparing the performance of the control of $\beta$ without (blue dash line) and with (red line) an anti-windup scheme.
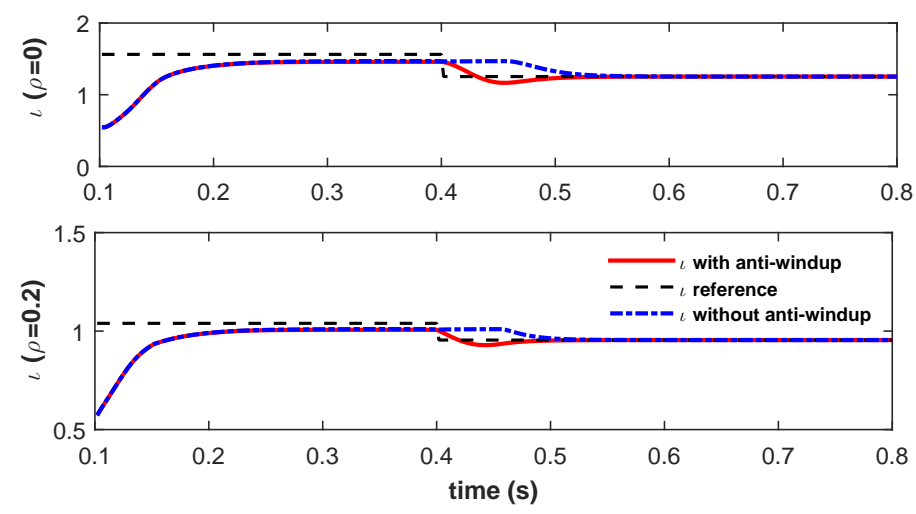

Figure C2: RAPTOR simulations comparing the performance of the control of $\iota$ with (blue dash line) and without (red line) an anti-windup scheme.

\section{Appendix C. Anti-windup implementation}

The powers of auxiliary antennas used in the tokamaks are subject to saturation. The saturation for the $i$-th actuator can be presented as:

$$
\operatorname{sat}\left(P_{E C, i}\right)=\left\{\begin{aligned}
P_{E C, i, \min } & \text { if } P_{E C, i}<P_{E C, i, \min } \\
P_{E C, i} & \text { if } P_{E C, i, \min } \leq P_{E C, i} \leq P_{E C, i, \max } \\
P_{E C, i, \max } & \text { if } P_{E C, i} \geq P_{E C, i, \max }
\end{aligned}\right.
$$

Integral windup can occur in loops where the actuator saturates and the controller has integral action. It occurs when the integral terms accumulate a significant error as a result of saturated integrators when the desired output is not achievable. This can lead to large overshoots and undesirable transients. To avoid windup, an extra feedback path is provided in the controller by comparing the difference between the output of the controller and the actuator output. Stability and anti-windup techniques for systems with saturated actuators can be found in [58, 59].

We applied anti-windup compensation by using the conditional integration (CI) technique that is presented in $[60,61]$. In the case where the saturation levels are 
known and where digital controller is used, this method can be effectively and easily implemented in the control. In this technique, the integral term is increased only when certain conditions are satisfied; otherwise it is kept constant. In the case of the auxiliary heating sources in tokamak plasma, the CI is applied such that the integral action is suspended when the actuators powers are saturated. In our case, the CI is calculated for each actuator separately. For the $z$ component, the integral term $\tilde{\mathcal{I}}_{\iota}$ is frozen when the actuators saturate as:

$$
\frac{\partial \tilde{\mathcal{I}}_{\iota}}{\partial t}=\left\{\begin{array}{cl}
0 & \text { if } \tilde{u} \text { saturates } \\
-\alpha_{I}(\rho) \tilde{z}-\lambda(t) \mathcal{I}_{\iota} & \text { otherwise }
\end{array}\right.
$$

In the case of the controller of $\beta$, the integral term $\tilde{\mathcal{I}}_{\beta}$ is frozen when the actuators are being driven into saturation:

$$
\frac{d \tilde{\mathcal{I}}_{\beta}}{d t}=\left\{\begin{array}{cc}
0 & \text { if } \tilde{u} \text { saturates } \\
K_{\beta, i} \tilde{\beta} & \text { otherwise }
\end{array}\right.
$$

The integration stops only when all the actuators are in saturation. Fig. C1 and Fig. C2 present the plots of $\beta$ and $\iota$-profile from a closed-loop RAPTOR simulation. In the time interval $t \leq 0.4 \mathrm{~s}$, the reference values of the outputs were selected such that the system actuators are forced to reach a saturation level. In this interval the integrator continues to accumulate the error if no anti-windup scheme is applied. After $t=0.4 \mathrm{~s}$ the target values are changed and the performance of the anti-windup technique can be tested. In the case when the anti-windup technique is not applied, there are delays in the controllers response to the change of the reference signal. From

these results it can be concluded that the simple CI technique successfully solves the controller windup problem.

\section{References}

[1] Taylor T S 1997 Plasma Physics and Controlled Fusion 39 B47

[2] Moreau D, Mazon D, Ariola M, De Tommasi G, Laborde L, Piccolo F, Sartori F, Tala T, Zabeo L, Boboc A et al. 2008 Nuclear Fusion 48106001

[3] Maljaars E, Felici F, De Baar M R, van Dongen J, Hogeweij G M D, Geelen P J M and Steinbuch M 2015 Nuclear Fusion 55023001

[4] Bribiesca Argomedo F, Witrant E, Prieur C, Georges D and Brémond S 2010 Model-based control of the magnetic flux profile in a tokamak plasma 49th IEEE Conference on Decision and Control (IEEE) pp 6926-6931

[5] Vu N M T, Nouailletas R, Lefèvre L and Felici F 2016 Control Engineering Practice 54 34-45

[6] Boyer M D, Barton J, Schuster E, Luce T C, Ferron J R, Walker M L, Humphreys D A, Penaflor B G and Johnson R D 2013 Plasma Physics and Controlled Fusion 55105007

[7] Boyer M D, Barton J, Schuster E, Walker M L, Luce T C, Ferron J R, Penaflor B G, Johnson R D and Humphreys D A 2014 IEEE Transactions on Control Systems Technology 22 1725-1739

[8] Laborde L, Mazon D, Moreau D, Murari A, Felton R, Zabeo L, Albanese R, Ariola M, Bucalossi J, Crisanti F et al. 2005 Plasma Physics and Controlled Fusion 47 155 
[9] Kim S H and Lister J B 2012 Nuclear Fusion 52074002

[10] Moreau D, Walker M L, Ferron J R, Liu F, Schuster E, Barton J E, Boyer M D, Burrell K H, Flanagan S M, Gohil P et al. 2013 Nuclear Fusion 53063020

[11] Barton J E, Boyer M D, Shi W, Wehner W P, Schuster E, Ferron J R, Walker M L, Humphreys D A, Luce T C, Turco F et al. 2015 Nuclear Fusion 55093005

[12] Barton J E, Besseghir K, Lister J and Schuster E 2013 Robust control of the safety factor profile and stored energy evolutions in high performance burning plasma scenarios in the ITER tokamak 52nd IEEE Conference on Decision and Control (Florence, Italy) pp 4194-4199

[13] Barton J E, Besseghir K, Lister J and Schuster E 2015 Plasma Physics and Controlled Fusion $\mathbf{5 7} 115003$

[14] Bribiesca Argomedo F, Prieur C, Witrant E and Brémond S 2013 IEEE Transactions on Automatic Control 58 290-303

[15] Gahlawat A, Witrant E, Peet M M and Alamir M 2012 Bootstrap current optimization in tokamaks using sum-of-squares polynomials 51st IEEE Conference on Decision and Controll (IEEE) pp 4359-4365

[16] Gaye O, Autrique L, Orlov Y, Moulay E, Brémond S and Nouailletas R 2013 Automatica 49 2795-2804

[17] Vu N M T, Lefèvre L and Nouailletas R 2015 IFAC-PapersOnLine 48482 - 487 ISSN 2405-8963 8th Vienna International Conferenceon Mathematical Modelling

[18] Bribiesca Argomedo F, Witrant E, Prieur C, Brémond S, Nouailletas R and Artaud J F 2013 Nuclear Fusion 53033005

[19] Maljaars E, Felici F, Blanken T, Galperti C, Sauter O, de Baar M, Carpanese F, Goodman T, Kim D, Kim S, Kong M, Mavkov B, Merle A, Moret J, Nouailletas R, Scheffer M, Teplukhina A, Vu N, MST1-team T E and TCV-team T 2017 Nuclear Fusion $\mathbf{5 7} 126063$

[20] Felici F, Sauter O, Coda S, Duval B P, Goodman T P, Moret J M, Paley J I, Team T et al. 2011 Nuclear Fusion 51083052

[21] Felici F, de Baar M and Steinbuch M 2014 A dynamic state observer for real-time reconstruction of the tokamak plasma profile state and disturbances American Control Conference (Portland,USA) pp 4816-4823 ISSN 0743-1619

[22] Felici F and Sauter O 2012 Plasma Physics and Controlled Fusion 54025002

[23] Hinton F L and Hazeltine R D 1976 Reviews of Modern Physics 48239

[24] Blum J 1989 Numerical Simulation and Optimal Control in Plasma Physics: With Applications to Tokamaks (Wiley Gauthier-Villars Series in Modern Applied Mathemat)

[25] Pereverzev G and Yushmanov P 2002 ASTRA automated system for transport analysis in a tokamak report ipp 5/98, Max-Planck-Institut für Plasmaphysik, Garching

[26] Sauter O, Angioni C and Lin-Liu Y R 1999 Physics of Plasmas 6 2834-2839

[27] Sauter O, Angioni C and Lin-Liub Y R 2002 Physics of Plasmas 95140

[28] Witrant E, Joffrin E, Brémond S, Giruzzi G, Mazon D, Barana O and Moreau P 2007 Plasma Physics and Controlled Fusion 491075 
[29] Mavkov B, Witrant E and Prieur C Distributed control of coupled inhomogeneous diffusion in tokamak plasmas IEEE Transactions on Control Systems Technology (to appear) URL https://doi.org/10.1109/TCST . 2017.2768039

[30] Wesson J and Campbell D 2011 Tokamaks vol 149 (Oxford University Press)

[31] Witrant E and Brémond S 2011 Shape identification for distributed parameter systems and temperature profiles in tokamaks 50th IEEE Conference on Decision and Control and European Control Conference (Orlando (FL),USA) pp 2626-2631

[32] Doyle E J, Houlberg W A, Kamada Y, Mukhovatov V, Osborne T H, Polevoi A, Bateman G, Connor J W, Cordey J G, Fujita T et al. 2007 Nuclear Fusion 47 S18

[33] Vu N T, Nouailletas R, Maljaars E, Felici F and Sauter O 2017 Fusion Engineering and Design $123624-627$

[34] Kong M, Sauter O, Blanken T, Felici F, Galperti C, Goodman T, Hogeweij G, Kim D, Kim S, Maljaars E et al. 2017 Real-time control of neoclassical tearing modes and its integration with multiple controllers in the tcv tokamak 44th EPS Conference on Plasma Physics (Belfast, Northern Ireland)

[35] Lyapunov A 1892 The general problem of the stability of motion (Kharkov Mathematical Society)

[36] Ljung L 1998 System identification (Springer)

[37] Luo Z H, Guo B Z and Morgül Ö 2012 Stability and stabilization of infinite dimensional systems with applications (Springer Science \& Business Media)

[38] Curtain R F and Zwart H 2012 An introduction to infinite-dimensional linear systems theory vol 21 (Springer Science \& Business Media)

[39] Åström K J and Hägglund $\mathrm{T} 2006$ Advanced PID control (ISA-The Instrumentation, Systems and Automation Society)

[40] Ziegler J G and Nichols N B 1942 trans. ASME 64 759-768

[41] Skogestad S 2001 Probably the best simple PID tuning rules in the world AIChE Annual Meeting, Reno, Nevada vol 77

[42] Grimholt C and Skogestad S 2012 IFAC Proceedings Volumes 45 11-22

[43] Bribiesca Argomedo F, Witrant E and Prieur C 2014 Safety Factor Profile Control in a Tokamak (Springer)

[44] Felici F, Blanken T C, Maljaars E, van den Brand H, Citrin J, Hogeweij D, Scheffer M, de Baar M R, Steinbuch M, Coda S et al. 2016 Real-time model-based plasma state estimation, monitoring and integrated control in TCV, ASDEX-upgrade and ITER 26th IAEA Fusion Energy Conference EX/P8-33

[45] Moret J M, Duval B, Le H, Coda S, Felici F and Reimerdes H 2015 Fusion Engineering and Design $911-15$

[46] Blanken T, Felici F, Rapson C, de Baar M and Heemels W 2018 Fusion Engineering and Design 12687 - 103

[47] Blanken T C, Felici F, de Baar M R, Heemels W P M H et al. 2015 Model-based reconstruction and feedback control of the plasma particle density in tokamaks 42nd European Physical Society Conference on Plasma Physics P2.151

[48] Felici F, Le H B, Paley J I, Duval B P, Coda S, Moret J M, Bortolon A, Federspiel L, Goodman T P, Hommen G et al. 2014 Fusion Engineering and Design 89 165176 
[49] Le H B, Felici F, Paley J I, Duval B P, Moret J M, Coda S, Sauter O, Fasel D, Marmillod P et al. 2014 Fusion Engineering and Design 89 155-164

[50] Krstic M and Smyshlyaev A 2008 Boundary control of PDEs: A course on backstepping designs vol 16 (Siam)

[51] Moura S J and Fathy H K 2013 Journal of Dynamic Systems, Measurement, and Control 135034501

[52] Boyer M D, Barton J, Shi W, Wehner W, Schuster E, Ferron J, Walker M, Humphreys D, Turco F, Luce T, Johnson R and Penaflor B 2014 IFAC Proceedings Volumes 471568 - 1573 ISSN 1474-6670 19th IFAC World Congress

[53] Ilhan Z O, Wehner W P and Schuster E 2016 Model predictive control with integral action for the rotational transform profile tracking in nstx-u 2016 IEEE Conference on Control Applications pp 623-628

[54] Wehner W, Barton J and Schuster E 2017 Combined rotation profile and plasma stored energy control for the diii-d tokamak via mpc 2017 American Control Conference pp 4872-4877

[55] Maljaars B 2017 Model predictive profile control and actuator management in tokamaks Ph.D. thesis TU Eindhoven, Eindhoven, The Netherlands

[56] Kaplan W 2002 Advanced calculus (Addison Wesley)

[57] Löfberg J 2004 YALMIP: A toolbox for modeling and optimization in matlab IEEE International Symposium on Computer Aided Control Systems Design (Taipei, Taiwan) pp 284-289

[58] Tarbouriech S, Garcia G, Gomes da Silva Jr J M G and Queinnec I 2011 Stability and stabilization of linear systems with saturating actuators (Springer Science \& Business Media)

[59] Bohn C and Atherton D P 1995 IEEE Control Systems 15 34-40

[60] Hanus R, Kinnaert M and Henrotte J L 1987 Automatica 23 729-739

[61] Hodel A S and Hall C E 2001 IEEE Transactions on Industrial Electronics 48 $442-451$ 